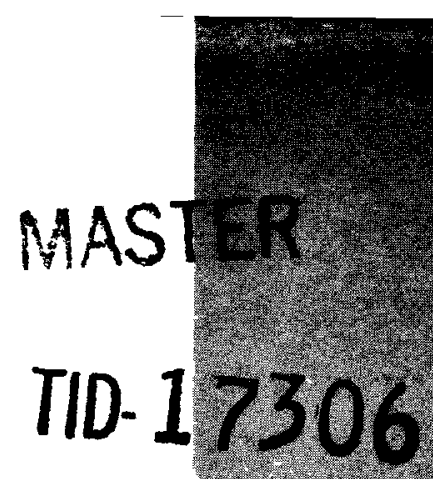

\title{
Application of \\ Nuclear Power Supplies to Space Systems
}

Eigma Corporation
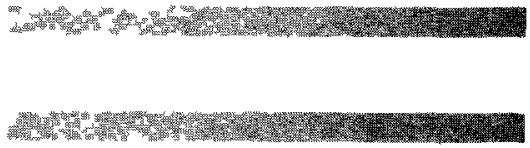

and
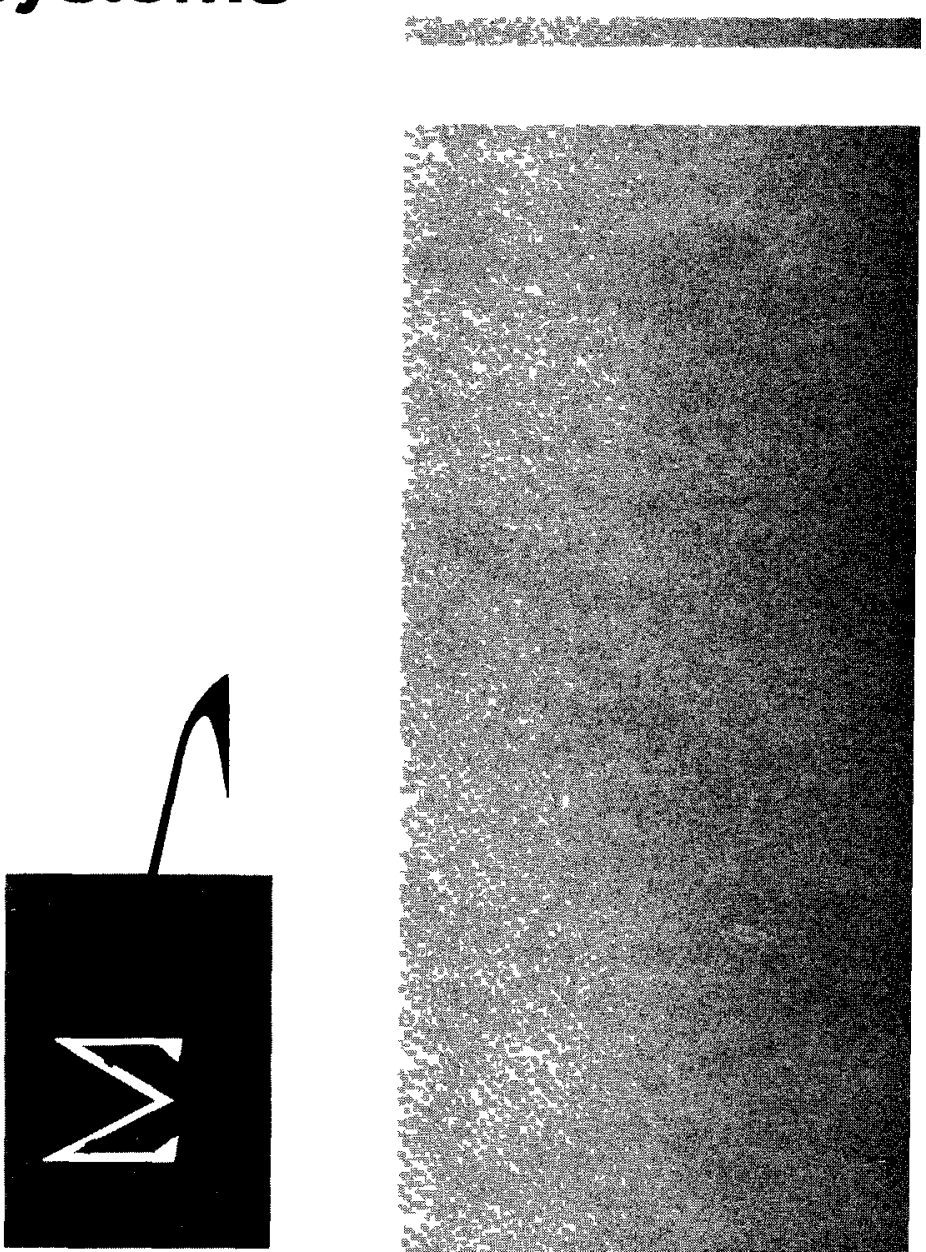

95 Main St. - Los Altos - Callfornıa 


\section{DISCLAIMER}

This report was prepared as an account of work sponsored by an agency of the United States Government. Neither the United States Government nor any agency Thereof, nor any of their employees, makes any warranty, express or implied, or assumes any legal liability or responsibility for the accuracy, completeness, or usefulness of any information, apparatus, product, or process disclosed, or represents that its use would not infringe privately owned rights. Reference herein to any specific commercial product, process, or service by trade name, trademark, manufacturer, or otherwise does not necessarily constitute or imply its endorsement, recommendation, or favoring by the United States Government or any agency thereof. The views and opinions of authors expressed herein do not necessarily state or reflect those of the United States Government or any agency thereof. 


\section{DISCLAIMER}

Portions of this document may be illegible in electronic image products. Images are produced from the best available original document. 


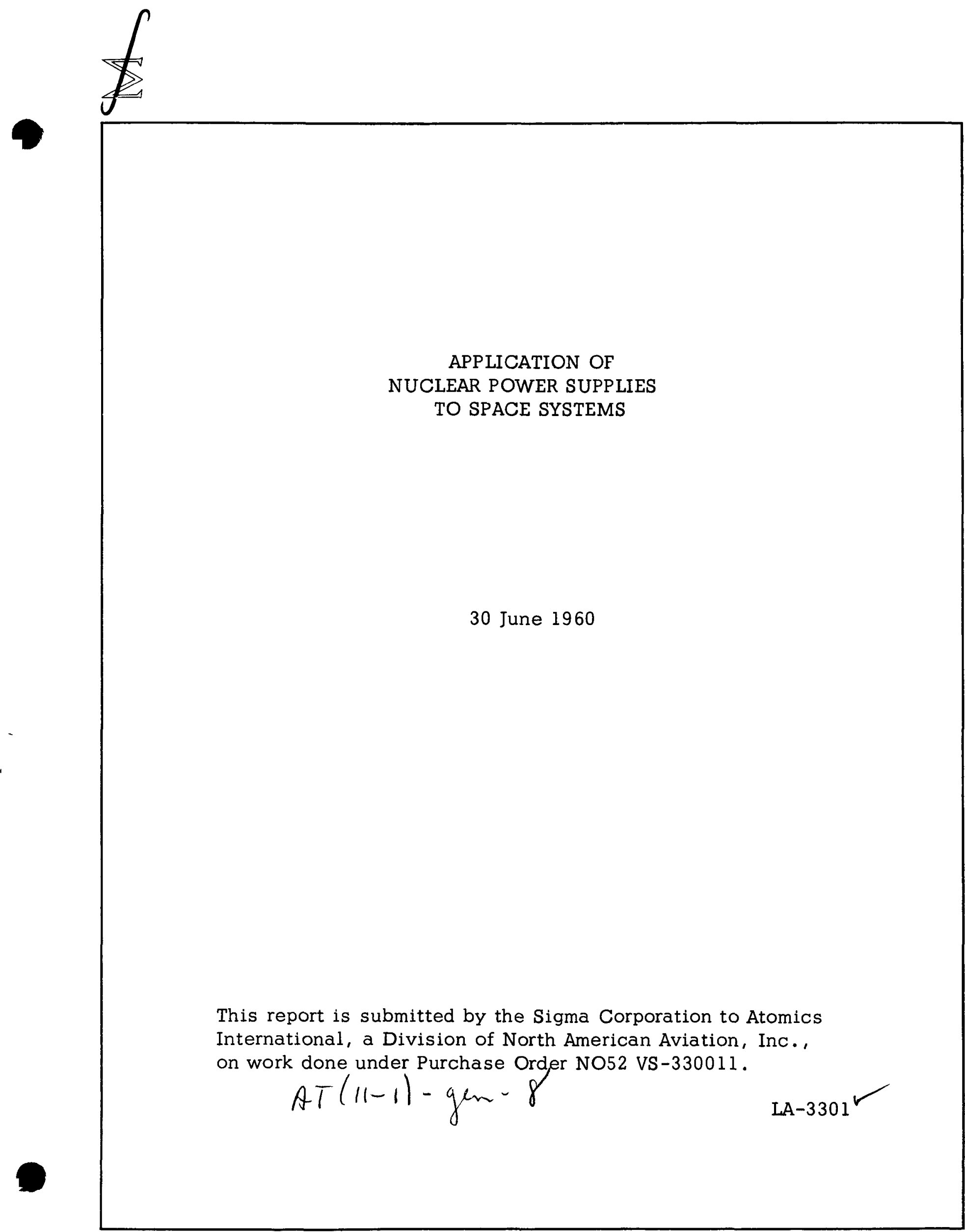


APPLICATION OF NUCLEAR POWER SUPPLIES TO SPACE SYSTEMS

Table of Contents

$\underline{\text { Section }}$

1.

1.1

1.2

1.3

1.3 .1

1.3 .2

1.3 .3

1.3 .4

2.

2.1

2.2

2.3

2.4

3.

3.1

3.2

3.3
Title

Introduction and Conclusions

Task Statement

The Approach

Conclusions

General Conclusions

Conclusions - SNAP 2

Conclusions - SNAP 8

Conclusions - SNAP 10

Space Flight Programs

Introduction

Scientific Space Exploration

The Military Space Program

Commercial Applications of Space Vehicles

Characteristics and Limitations of SNAP

Introduction

Space System Characteristics of SNAP Developments

Comparison of SNAP and Solar APU $\underline{\text { Page }}$ 
Section

4.

4.1

4.2

4.2 .1

4.2 .2

4. 2.3

4.2 .4

4.2 .5

4.3

5 。

5.1

5.2

6.

6.1

6.2

Appendices

A

B

C
Title

Page

Applications of Nuclear Power Supplies

22

Introduction

22

Applications of Current SNAP Units

22

Competitive Position of Present SNAP Units

22

The Scientific Space Program

23

The Military Program

Commercial Satellite Applications

Chronological Development of SNAP and NPS Applications

Applications for Future Nuclear Power Supplies

Design Integration

38

Corfiguration and Installation

Environmental Effects

System Integration

45

Agena B

Other Vehicles

Agena B Flight Test Program

Nuclear-Electric Propulsion

Commercial Communications Satellite

60 


\section{LIST OF FIGURES}

Fig. 1

Fig. 2

Fig。 3

Fig。 4

Fig。 5

Fig。6

Fig. 7

Fig。 8

Fig。9

Fig. 10

Fig. 11

Fig。 12

Fig。B-1

Fig. B-2

Fig。C-1

Fig. C-2
Major Vehicle Laurchings Anticipated by NASA

Growth of Payload Launching Capability

Scientific Space Program (NASA)

Military Space Program Vehicle Launchings

Space System Characteristics of SNAP Developments

Generalized Comparison of SNAP and Solar APU System Weight

Comparative Illustration of SNAP and Solar APU System Availability

Comparative Illustration of SNAP and Solar APU System Cost

Summation of Space Program Applications of SNAP Developments

Growth in Payload Capability

Growth in Auxiliary Power Requirements

Estimated Power Requirements and SNAP Operational Availability

Projected Specific Impulse vs. ThrustWeight Ratios of Various Propulsion Systems

Characteristics of a Cesium-Diode Energy Converter

Atlantic Leg of a Commercial Communications Satellite System

Trainable Antenna Cost for Ground Installation vs. Radiated Power 


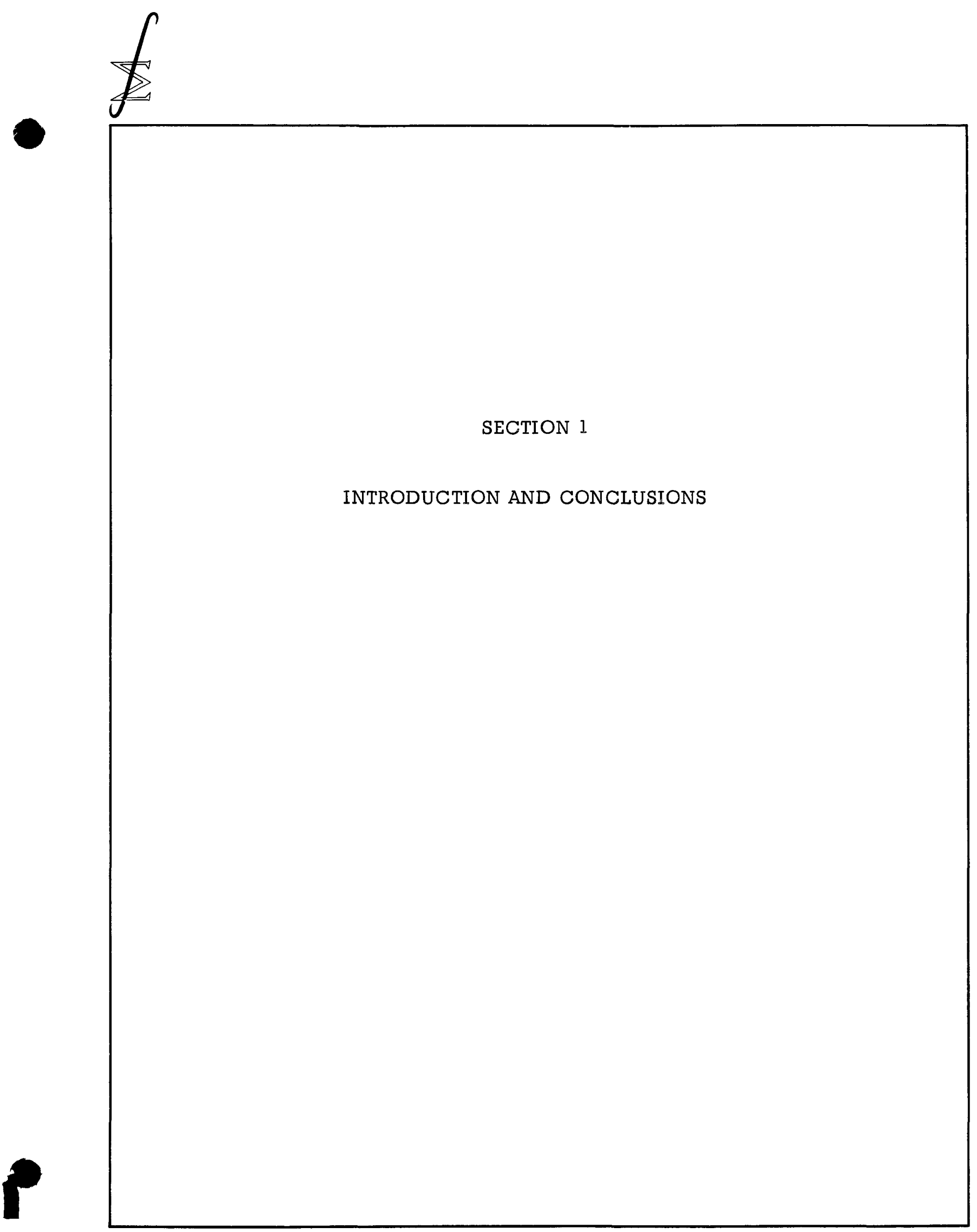

Sigma Corporation 


\section{INTRODUCTION AND CONCLUSIONS}

This report covers work performed under Atomics International Purchase Order NO52 VS-330011 and in accordance with study objectives specified in a letter from J.R. Wetch to R. M. Salter dated 16 April 1960. The study objectives were defined in terms of four primary task groups which are listed in the following section.

\subsection{Task Statement}

Task I - To ascertain what useful satellite and/or space missions may be accomplished with the SNAP 2 , 8 and 10 power systems within the 1960-1970 time period. Particular emphasis should be given to peaceful applications as opposed to strictly military objectives.

Task II - To delineate useful satellite and/ or space missions which would most profitably employ nuclear power sources and to establish the power requirements and time periods in which such missions would be feasible. Both military 
and commercial applications should be considered.

Task III - To ascertain what restrictions are imposed upon various payloads as a result of having a nuclear power system on board due to configuration, radiation, hot radiator, etc. To scope as far as possible radiation tolerances and sensitivities, temperature capabilities and heat dissipation requirements of the specific payloads delineated in Tasks I and II。

Task IV - To investigate the general vehicle integration and installation requirements of the SNAP 2 and 10 power units from an operational point of view. Particular emphasis should be given to defining the limitations imposed on these power systems by the specific vehicles and missions that are considered. The establishment of structural requirements and limitations of various geometrical configurations will be considered. This analysis will be limited to 
missions in the 1960-1970 time period. The Atlas-Agena B satellite vehicle will be employed as a datum for vehicle systems, although some look at the Atlas-Centaur and Saturn applications will be made.

\subsection{The Approach}

The approach to the study has been to first define, insofar as possible at this time, the space flight programs of this country. In this respect both formal and informal definitions of scientific, military and commercial applications of space flight have been examined. The outgrowth of this examination has resulted in defining a reasonably large group of missions and applications which can, when taken in toto, be considered to be the "probable space flight program of this country for the 1960-1970 time period".

Independent of the definition of the space flight program, the capabilities and limitations of SNAPs now in development and, in general, capabilities and limitations of SNAP-type systems which may be projected in future designs have been examined and compared with other types of APU systems.

A correlation of APU requirements associated with the space flight program and the capabilities and limitations of present and future SNAP systems results in the definition of some specific missions and applications where there is clearly a compelling need for SNAP units. The same correlation also results in 
the definition of some specific missions where the SNAP system is strongly competitive with solar APU systems and the decision will rest with factors which are not yet clearly defined. There is a final category of specific missions where the correlation of requirements and capabilities and limitations clearly indicates, either for technical or developmental time phasing reasons, that SNAP units are not applicable.

During the study period, results of a preliminary nature, concerning the applicability of SNAP units to commercial systems and, in particular, to a commercial communications satellite system were furnished as was also a report on the applications of SNAP 2, 8 and 10 units to operational satellite systems in the 1960 to 1970 time period. These preliminary results are incorporated in this more comprehensive study.

In addition to the general study leading to the applications of SNAP to space missions, the specific task of integrating SNAP 2 and SNAP 10 into a flight test package for testing on the Agena vehicle was undertaken. Because of the complex nature of this latter task, only a general treatment has been given in this report in a manner which follows and is correlated with the foregoing study of missions and applications. A "first look" has also been taken at the integration problem with respect to AtlasCertaur and Saturn systems.

\subsection{Conclusions}

1.3.1 General Conclusions.

The study has indicated that during 
the latter part of the 1960-1970 decade, power requirements for space vehicle APU will be considerably larger than they will be during the early part of the decade. These requirements will probably be from 1 to 5 kilowatts for unmanned vehicles and from 5 to 50 kilowatts for manned vehicles. These are APU requirements and do not include requirements for power which may be associated with the use of electric propulsion which will require power from 50 to 500 kilowatts and greater. SNAP units for the generation of power below 5 kilowatts will be in strong competition with solar cell APU systems. The decision to use one or the other of these two systems will result from an overall study of the total system in which the APU is to be used and in many cases economic factors (including reliability) and early availability will strongly influence the selection of the APU.

Where power requirements are greater than 5 kilowatts, it would appear at this time that SNAP units are clearly the desirable choice, both from the standpoint of technical desirability and economy of development and production.

In a minimal sense both SNAP 2 and SNAP 10 are required to demonstrate the feasibility and operability of SNAP power in orbiting vehicles. Future development work on these units should be oriented to increase the maximum power output of both of these systems to place them into a more favorable competitive position with solar supplies.

The present development time scale 
of SNAP units and lack of demonstrated operability in orbit has resulted in a situation where system designers tend to resist the use of SNAP units. This resistance can be expected to continue until orbital demonstrations of SNAP operability have become a reality. The present time scale for flight testing SNAP R \& D units seems to be unduly long and should be shortened.

It is, therefore, the general conclusion that emphasis on present development work should be placed on an early demonstration of orbital operability of SNAP. Future development should be aimed at fulfillment of high power requirements for APU and electric propulsion use. This development should take into account the fact that there will be need for its utilization in manned vehicles.

\subsubsection{Conclusions concerning SNAP 2 .}

The outcome of early flight tests

must be satisfactory before system designers will undertake to make decisions in favor of SNAP 2 in place of solar APU units. If, then, the reliability and lifetime of SNAP 2 is highly favorable, there is a certain probability that system designers will be encouraged to choose SNAP 2 in place of solar systems for complex military payloads and for payloads such as a comsatellite system where the higher power available and resultant advantages in design simplification would provide economic justification for the use of a SNAP unit. An increase in the electrical power output of SNAP 2 to the 5 kilowatt level would permit this unit to claim the market for increasing APU power in the latter 
half of the decade, in addition to holding a competitive position to solar units in meeting lower (1-3 kilowatt) power requirements.

$$
\text { 1.3.3 Conclusions regarding SNAP } 8 \text {. }
$$

The power available from SNAP 8 is

sufficient to provide for the utilization of electric propulsion for unmanned space missions. This application alone will provide a sizable market for SNAP 8 units.

In addition the possibility of a high power, long-lived communications satellite system for commercial intercontinental traffic, and the power demands of the earth satellite base and lunar bases will require nuclear power supplies at electrical output levels such as available from the SNAP 8 reactor. Development of this unit should include consideration of the specialized conversion techniques and configurations that may be needed to best serve these applications.

$$
\text { 1.3.4 Conclusions regarding SNAP 10. }
$$

The low power available from SNAP 10

places it in a regime where it is in a weak competitive position with solar cells. It seems likely that systems designers would not choose to use SNAP 10 unless a unique technical requirement appears. In the case of a Midas early warning satellite such a requirement might occur if a decision were made to harden the satellite against the effect of nuclear bursts designed to destoy solar cell APU systems and thus cause fallurs of the vehicle. Increases in the maximum power output of this type unit to $1-1.5$ kilowatt appear warranted, despite 
inherent weight penalties, to better serve the strong preference in system design for static conversion units. The increased payload capacity of the booster and vehicles systems which are programmed to be in time phase with such static units permit acceptance of the weight disadvantage in favor of the benefits to be derived. 


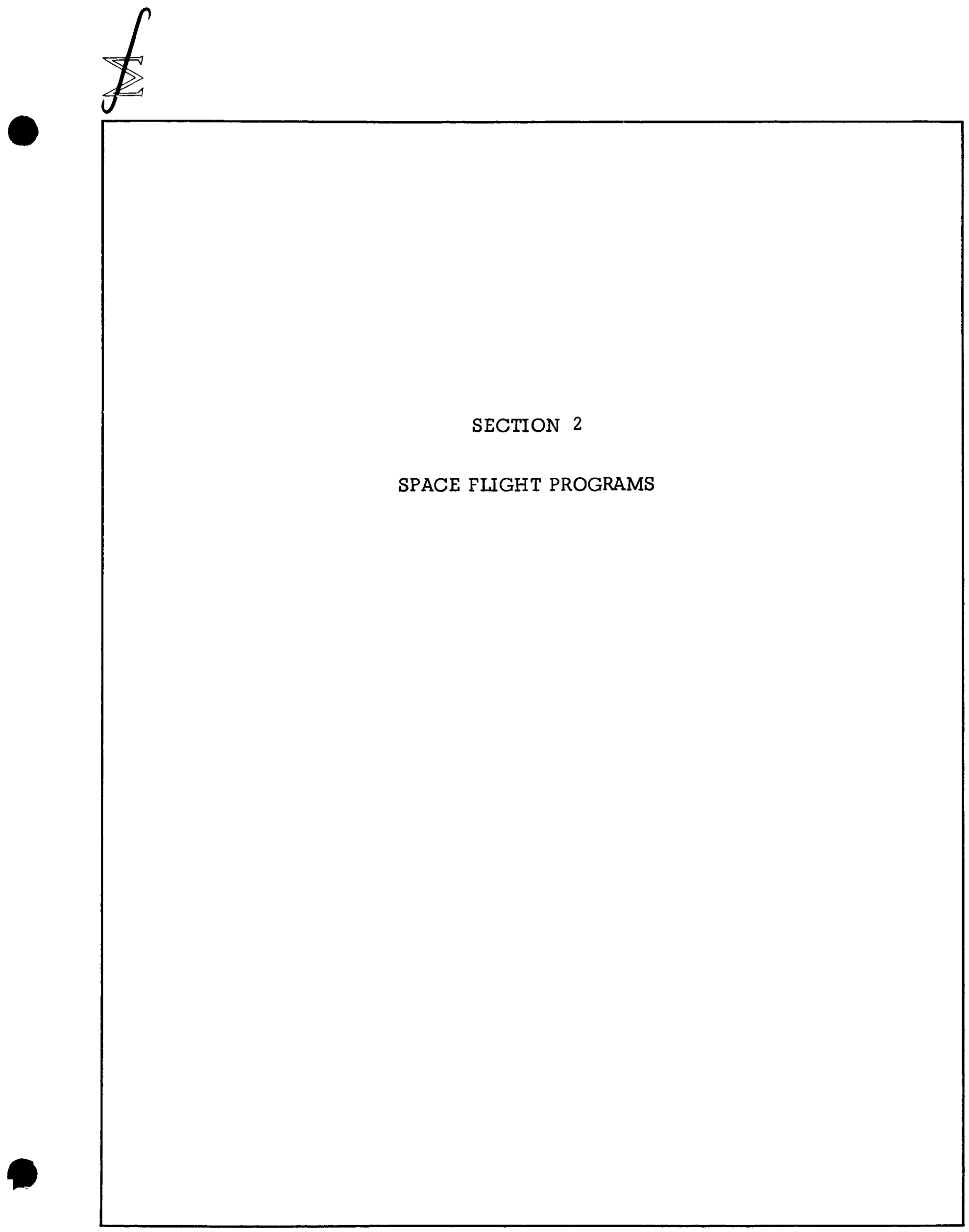




\subsection{Introduction}

Requirements for SNAP usage must stem from basic space program requirements and a definition or anticipation of these is dependent upon an overall definition of the total U.S. space program in being and anticipated. However, there is not now and probably never will be a formal integrated space program planned and administered as such in the U.S. Therefore, in order to scope the total space effort in being and anticipated, the various individual programs in being and planned must be compiled and analyzed to provide a reasonably comprehensive picture which can be called the U.S. Space Program.

The specific programs which are in being and those which are anticipated can be grouped into three major categories: Scientific, Military and Commercial. The category of scientific space missions is essentially synonomous with the NASA program and will be examined as such. The military program with minor exceptions is and will probably continue to be a program of activities planned and administered by the Air Force. The specific nature of commercial applications of space vehicles can only be dimly perceived at this time. However, the general concept of a commercial communications satellite seems to provide at least one concrete example of a commercial application which will occur within a decade.

There is a strong tendency to speculate about and enumerate a large number of possible space missions and applica- 
tions. This tendency has been purposely curbed in this study as tending to be misleading since the economy of the nation must of necessity limit the scope of actual space activities to a small percentage of those which are technically possible and even strongly desirable. The activities and programs which are analyzed here are those which are in being and expected to continue, and those which appear to have a high probability of becoming active within the fiscal and political framework foreseen for the next several years.

\subsection{Scientific Space Exploration}

The NASA has within recent months defined a general program of scientific space exploration which it intends to follow during the 1960-1970 period. The foundation of the program is established by the types and numbers of launching vehicles which will be available. Anticipated NASA vehicle launchings for the period are shown in Fig. 1. At this time the payload stages to be flown with these vehicles are only vaguely defined for the period beyond 1962. However, it is reasonable to assume that these stages will be configured to utilize the available payload capability of the launch vehicle. The growth in payload capability, illustrated by Fig. 2, thereby gives some insight into the probable sizes of payload stages which may evolve over the decade.

The current program of NASA, as reflected in the following categories derived from FY 1961 budgetary presentations, serves as a point of departure in describing the national 
MAJOR VEHICLE LAUNCHINGS

ANTICIPATED BY NASA

VEHICLE

$$
\begin{array}{lllllll}
62 & 63 & \frac{\text { FISCAL YEAR }}{64} 65 \quad 66 & 67 & 68 & 69
\end{array}
$$

TOTAL

Scout

Thor-Delta

$\begin{array}{llllllll}6 & 6 & 6 & 6 & 6 & 6 & 6 & 6\end{array}$

48

Thor-Agena B

Atlas-Agena B

$\begin{array}{llll}3 & 4 & 5 & 6\end{array}$

Atlas-Centaur

54

Saturn

22

Nova-Type

$\begin{array}{lllllllll}22 & 22 & 25 & 28 & 28 & 28 & 29 & 30 & 212\end{array}$

Fig. 1 


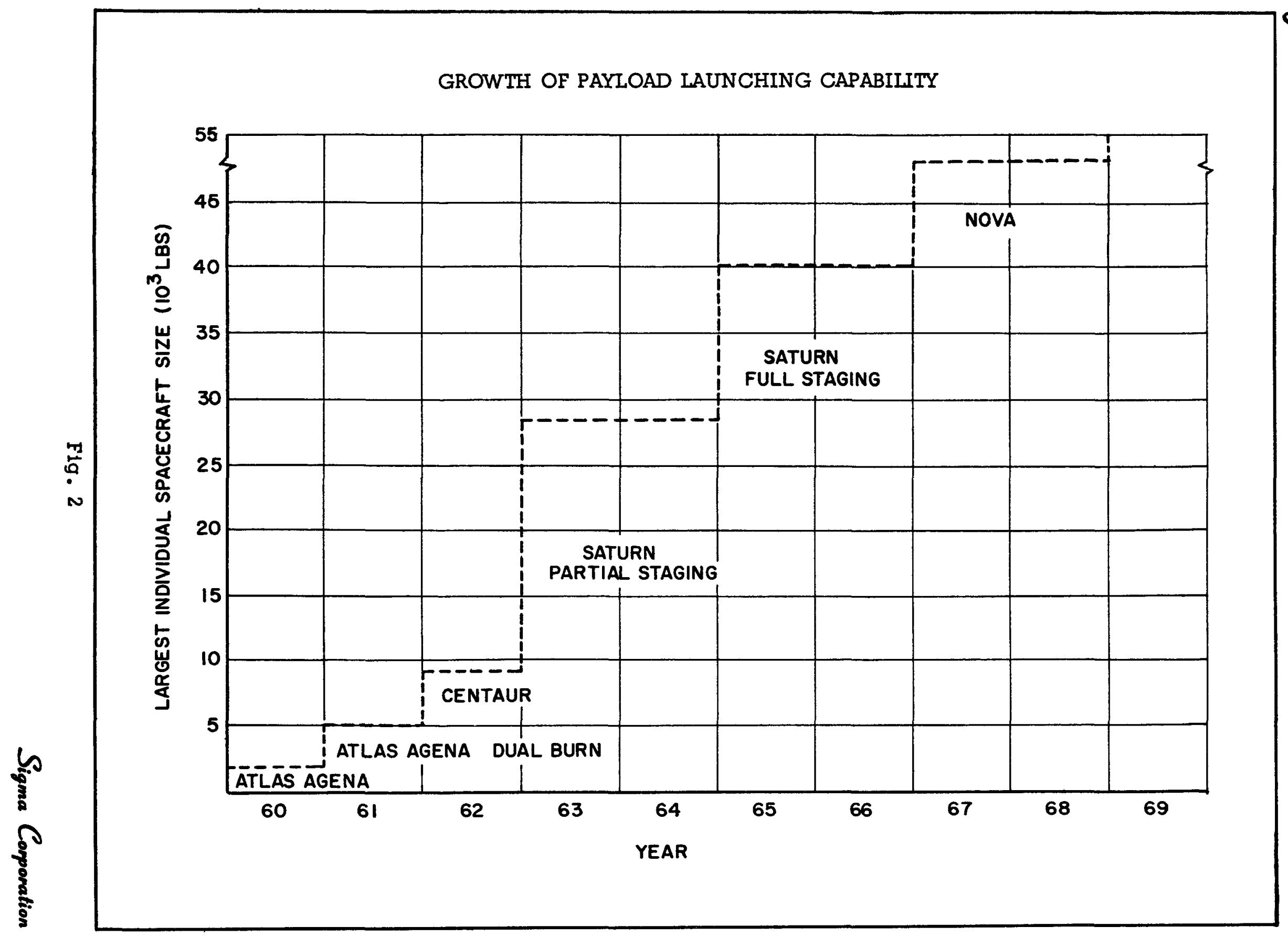




\section{$\sum$}

scientific space program of the next decade.

NASA Research and Development Programs

FY 1961

Space Propulsion Technology

Solid rockets

Liquid rockets

Nuclear systems

Space power systems

Electric Propulsion

Vehicle Systems Technology

Tracking and Data Acquisition

Satellite Applications

Meteorological - Tiros

Communications - Echo

Manned - Mercury

Space Investigation

Sounding rockets

Scientific data satellites

Lunar and Planetary explorations

Vehicle Development

Scout

Delta

Centaur

Saturn

The heterogeneity of the subjects shown above

clearly demonstrates the youth of our national space program. 
Dollar allocations associated with the se groupings show clearly the emphasis that is being placed on the Mercury program and development of the Saturn vehicle, the two key projects in establishing and extending U.S. programs for man in space. Recent NASA releases describing its aspirations over the next ten-year period employ a new grouping of research program categories. These programs, as described in the following paragraphs, give evidence of reorientation during the next few years to more logically reflect the scientific space program as the principal objective of NASA.

The Atmos pheric Research Program is dedicated to the determination of the chemical constitution and atmospheric behavior (pressure, density, temperature, etc.) of the earth, the sun, the moon and the planets. Earth atmospheric research data is being collected currently through employment of sounding rockets and ballistic vehicles. In addition, earth meteorological science is being served by collection of data from the Tiros vehicle. Upper atmosphere soundings with Scout will essentially complete the requirements visualized at present. Solar atmosphere research will require penetration and impact trajectories employing such vehicles as Atlas-Able, Atlas-Delta and AtlasAgena B. For the lunar program, 4-500 pound impact payloads such as may be delivered using the Agena B would first be required, and later up to 4000 pound impact payloads may be delivered by the Saturn vehicle. For planetary atmospheric research early impacts, near misses and planetary orbits may be attempted using Atlas-Centaur, later Saturn with upper stages 
powered either by conventional propulsion systems or electric propulsion, may be employed, both for orbits and impacts.

The atmospheric and ionospheric research programs would appear to be the first efforts to be completed in the next decade. The similarity of trajectories required to obtain the desired data indicates that the sensory devices for collecting data for both of these programs will share payload space on the same vehicles. In addition, these program payloads will probably be joined by measurement equipment of the energetic particles program described below.

The ionospheric research program will have as its objective the determination of sources, natures and spacial distributions and behavior of the ionized regions of the solar system. Present data on the earth's ionosphere will be supplemented by Project Echo balloon satellite communication tests . Earth ionospheric top sounding may be performed by an earth satellite vehicle of the Agena $B$ type which may in addition serve as a space to earth communication relay station for collecting comparative communications data on transmissions both from space probes and lunar impacted radio transmitters. The later flights would require either Agena B or Atlas-Centaur vehicles.

The Energetic Particle Research Program is selfexplanatory in its purpose. Data for this program may be obtained from earth satellite instrumentation in addition to probe flights in the Van Allen regions followed by lunar satellites. Later efforts will include lunar observatories and the collection of data from 
planetary orbits. This program will probably be served by payload sharing in Centaur, Agena B and Saturn vehicles as described in the foregoing paragraphs.

The Electric and Magnetic Fields Research Program and the gravitational fields research effort will emply highly eccentric earth, lunar and planetary orbits in addition to early probes and sounding rocket flights. Both the Thor and AtlasAgena B vehicles and the Centaur will be required for the long period orbital station requirements.

The Gravitational Fields Research Program, in addition to sharing of payload capacity on the electric and magnetic fields flights identified above, is visualized to include surface explorations after 1968 on the moon, which activity will require use of the Saturn vehicle. In addition, exploration of the gravitational fields of other regions of the solar systems, and perhaps other galaxies, are envisioned and will, if performed, require extremely high power levels and long power supply life to support electric propulsion units and long range communications equipment。

The Astronomical Research Program has as its objective the establishment of a lunar astronomical laboratory for solar and stellar observation. The lunar base required to meet this objective may be established and served by Saturn-type vehicles staging through an earth satellite station. Power requirements on both the satellite station and the lunar base may best be satisfied by nuclear power supplies. The lunar base observatory is 
preferred to the earlier satellite station astronomical laboratory since, in addition to being free of the earth's atmospheric filtration of ultra-violet, the lunar station will also benefit from earth shielding of the base from sunlight during stellar observations.

The Bio-Sciences Research Program centers upon the manned earth satellite station which will serve as a long term observatory for bio-sciences in space. This mission, as indicated before, requires the Saturn vehicle. To detect or deny the presence of biological life on the other planets, a series of reconnaissance research flights is visualized employing Centaur and Saturn vehicles equipped with visual and IR sensing payloads. The Mercury project is not included as a part of the bio-sciences program, but instead is considered a means to the end of establishing man in both satellite station and lunar laboratories.

Fig. 3 summarizes in tabular form the major portion of the scientific space program and identifies clearly the work horse role which will be played by the Agena, Centaur and Saturn vehicles. Despite the fact that joint payloads will be employed when possible in obtaining data for each of the several research programs, it can be seen from this Figure and from the planned schedule of NASA launchings that the scientific space program will be the most meaningful U.S. space undertaking over the next decade.

2.3 The Military Space Program

The military space program, for purposes of this study 
$\sum$

SCIENTIFIC SPACE PROGRAM (NASA)

1960-1970

THOR

AGENA B

Ionospheric

Space-earth

Communications Relay

Top Soundings

Earth Orblt

\section{Energetic Particles \\ Earth Orbits}

Electric \& Magnetic

Fields

Earth Orbit
ATIAS

AGENA B

Lunar Atmos pheric

Orbit \& Impact

Solar Atmospheric

Orbit

Lunar Ionospheric

Lunar-earth

Communications

Soft Impact

Communication Relay

Top Soundings

Energetic Particles

Lunar Orbits

Lunar Observatory

Electric \& Magnetic

Flelds

Earth Orbit

Electric \& Magnetic

Flelds

Lunar Orbit

Electric \& Magnetic

Fields

Lunar Orbit

Electric \& Magnetic

Fields

Planetary Orbits

Gravitational Fields

Earth Orbits

Lunar Orbits
ATLAS

CENTAUR

Planetary Atmospheric Orbits

Ionospherlc

Lunar-earth

Communlcations

Soft Impact

Lunar Ionospheric

Planetary Ionosphertc

Energetic Particles

Planetary Orbits

Planetary Impacts
Electric \& Magnetic Fields

Lunar Explorations

Electric \& Magnetic Flelds

Galactic Probes Gravitational

Astronomy
Lunar Observatory
Soft Impacts \& Return
Satellite Station
Earth Orbit
Lunar Staging
Blo-Science Labs
Planetary
Reconnaissance
(V1sual \& IR)

Fig. 3

Blo-Sclences

Planetary

Reconnalssance

(Visual \& IR) 
has been described and evaluated on an unclassified basis. Considerations of basic program purposes together with logical system configurations and economic values have been used to establish the probable military program for the 1960-1970 decade.

The WS 117L program which has been in progress through study and development efforts for the past decade will continue to be one of the most important military operations in connection with space activities during the next decade. The 117L program is essentially composed of three portions: the Midas program, the Samos program and the Discoverer program. The Samos program is aimed at establishing a network of surveillance and reconnaissance satellites capable of gathering data in the visual spectrum. The Midas program is aimed at establishing a network of satellites capable of detecting ballis tic missiles during the launching phase with infra-red instrumentation. The Discoverer program consists of a series of satellite firings to provide data for advanced research and development.

In addition to the $117 \mathrm{~L}$ program, a major communications satellite program has been established which is aimed at providing a network of communications relay stations on 24hour satellite orbits to provide a greatly increased long distance military communications systems.

The Dyna-Soar program may eventually become an integrated part of the overall military space program. The advanced techniques of manned orbital flight and re-entry which will be pioneered by this program will supplement the work now being 
done in the Mercury program. The sum total of the manned space flight effort seems to be aimed at supplying the necessary technology for establishing a manned space station which can then be used for manned exploratory flights and eventually establishment of manned bases on the moon and planets.

In the 1960-1970 decade it is estimated that the probable military space program of launchings will be similar to that shown in Fig. 4. This program of launchings is sufficient to establish and operate the Samos, Midas and com-satellite systems and will, in addition, provide a research and development effort to support programs of manned space activity in the 19701980 period.

As indicated previously, the forthcoming national programs of man in space fall within the cognizance of NASA. Both politically and functionally (over the next decade), it would appear desirable to maintain this identification, but at the same time it is necessary to establish our initial national military capability to claim, counter-claim and defend, if necessary, rights in space. Therefore it is anticipated that DOD, in particular the USAF, will participate closely with NASA in evolving our manned space capability, including provision of personnel, planning, base and logistic support and funds. As such the manned space activities of the next decade may be considered a part of the military space program, an increasingly important part which will reflect itself in the future posture of the Air Force. 


\section{MILITARY SPACE PROGRAM}

VEHICLE LAUNCHINGS (UNCLASSIFIED ESTIMATE)

$$
\underline{1960-1970}
$$

PROGRAM

FISCAL YEAR

TOTAL

$$
\begin{array}{llllllll}
62 & 63 & 64 & 65 & 66 & 67 & 68 & 69
\end{array}
$$

Samos

$\begin{array}{llllllll}6 & 12 & 12 & 12 & 12 & 12 & 12 & 12\end{array}$

Midas

$\begin{array}{lll}6 & 12 & 10\end{array}$

8

5

55

Com Sat (24-hr)

$2 \quad 2$

Military $R \& D$

(Discoverer, Dyna-Soar, etc.)

\section{$\begin{array}{llllllll}24 & 29 & 28 & 26 & 23 & 23 & 24 & 26\end{array}$}

This estimate of military space vehicle firings has been based on unofficial and unclassified reports of NASA and DOD budgets and Midas and Samos system descriptions contained in Aviation Week Magazine dated Jan. 28, 1960, Fe. 8, 1960, May 2, 1960 and May 30, 1960. No classified information was used in the preparation of this estimate. The authors have stringently guarded against educated selection of published data to preclude compromises of retained security information from previous classified work.

Fig. 4 


\subsection{Commercial Applications of Space Vehicles}

It is likely that by the late 1960 's some of the overall system technology which has been developed by military and scientific space applications can be diverted to commercial systems. Development of commercial applications is, of course, dependent upon the establishment of economic need. At this time it appears that the only justifiable commercial system utilizing space vehicles would be a communication relay network. Studies have shown that component cost and operating cost may become such that a satellite system can provide cheaper and more reliable relay stations than any other conceivable means, thereby providing strong economic incentive for the establishment of a commercial communications satellite network. The concept of this type of system is covered in greater detail in Appendix C. 


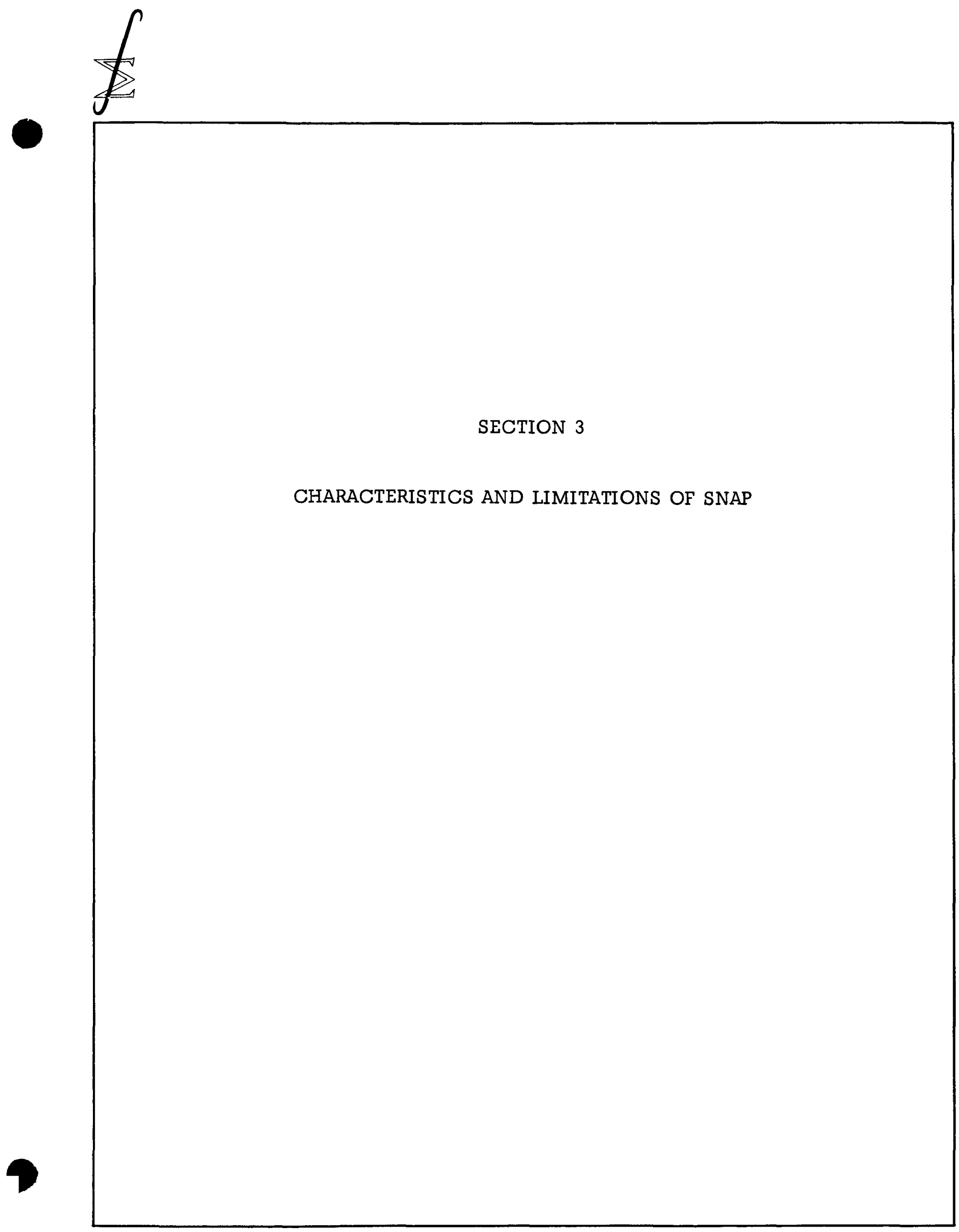

Sigma Corporation 


\subsection{Introduction}

The characteristics and design features of SNAP units now being developed have been covered in references 1,2 and 3. Reference 1, as well as many other published papers, has also demonstrated that systems having an expected operating life greater than a few weeks must be supplied with power from nuclear or solar APU. The information contained in the referenced documents will be assumed to be known and will not be repeated in this report. The following section is based on this assumption.

3.2 Space System Characteristics of SNAP Developments

The SNAP program has resulted in an outgrowth of Rand studies which formed the background of the 117L program. As such the SNAP program has been largely geared to $117 \mathrm{~L}$ program requirements and specifically the characteristics of SNAP 2 evolved from early 117L system studies. During these early studies there was insufficient technical knowledge to make a choice between nuclear power supplies and solar power supplies. However, the advent of Sputnik with the resulting speed-up of U.S. space programs and the low payload capability of existing U.S, boosters resulted in the early development and flight test of solar power supplies. This demonstrated capability of solar power supplies, together with other desirable aspects such as a relatively high degree of freedom pertaining to design flexibility, and a lack of undesirable characteristics such as attendant nuclear 
radiation form strong incentives for system designers to select solar APU supplies at an early point in their system design.

SNAP systems have therefore been examined in this context to determine whether or not they can compete successfully with solar supplies and if so, in what regime of characteristics this will occur. Fig. 5 contains some gross space system characteristics of SNAP developments which are particularly useful to the Space System Designer.

3.3 Comparison of SNAP and Solar APU

The Space System Designer will be constrained in an initial evaluation of possible power supplies to think in terms of availability, payload weight allowance, design flexibility and cost. The approximate relationship of SNAP and solar supplies with respect to weight, availability and cost are shown in Fig. 6, 7 and 8 , respectively. Design flexibility is less subject to such generalized and simplified treatment and must be considered for specific cases. The integration of SNAP units in Agena vehicles is covered in a later section.

As indicated in Fig. 6, where weight is used as the measure of merit, solar supplies are clearly superior to SNAP below 1 kilowatt. In the power regime from 1 to 5 kilowatts solar supplies and SNAP are competitive with SNAP systems becoming superior above 5 kilowatts for unmanned systems. Manned systems with their attendant shielding requirement cause the power regime to be extended to 15 kilowatts before SNAP becomes superior. This, of course, assumes that all of the shielding weight 
SPACE SYSTEM CHARACTERISTICS

OF SNAP DEVELOPMENTS

CHARACTERISTIC

Availability Date for

Mission Use

Power Output

Approx. SNAP Wt.

(without shield)

Approx. Shield

Weights for

Hard Electron Tubes

Present Type

Transistors

$\operatorname{Man}$
SNAP DESIGNATION

SNAP $10 \quad$ SNAP 2 SNAP 8

Sept $63 \quad$ Sept $64 \quad$ Jan 66

$300+$ watts $3 \mathrm{KW} \quad 30 \mathrm{~kW}$

$300 \mathrm{lbs}$. $600 \mathrm{lbs} . \quad 1500 \mathrm{lbs}$.

0

0

$35 \mathrm{lbs}$.

$250 \mathrm{lbs}$. $250 \mathrm{lbs} .320 \mathrm{lbs}$.

$2000 \mathrm{lbs} .2200 \mathrm{lbs}$.

Fig. 5 

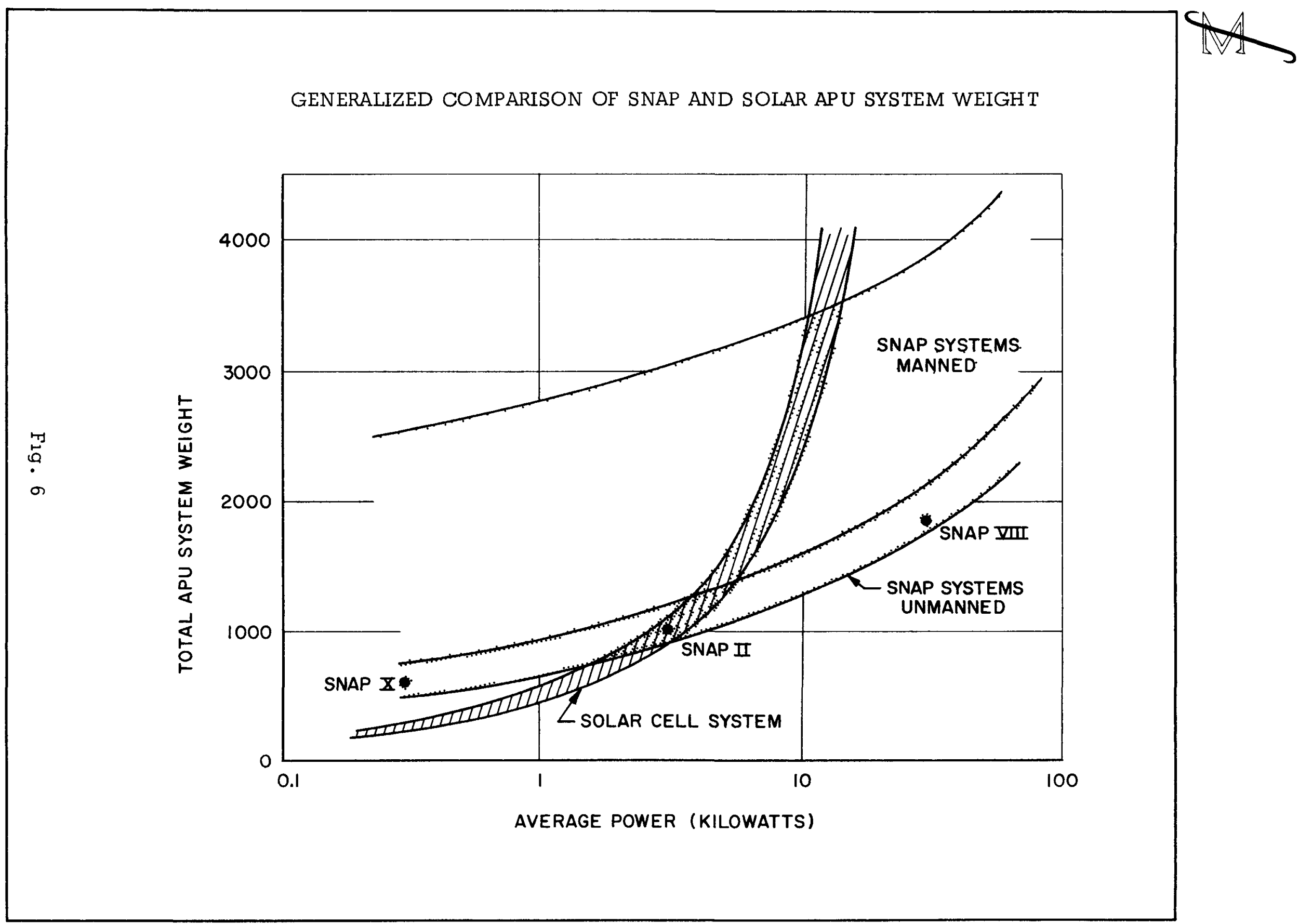


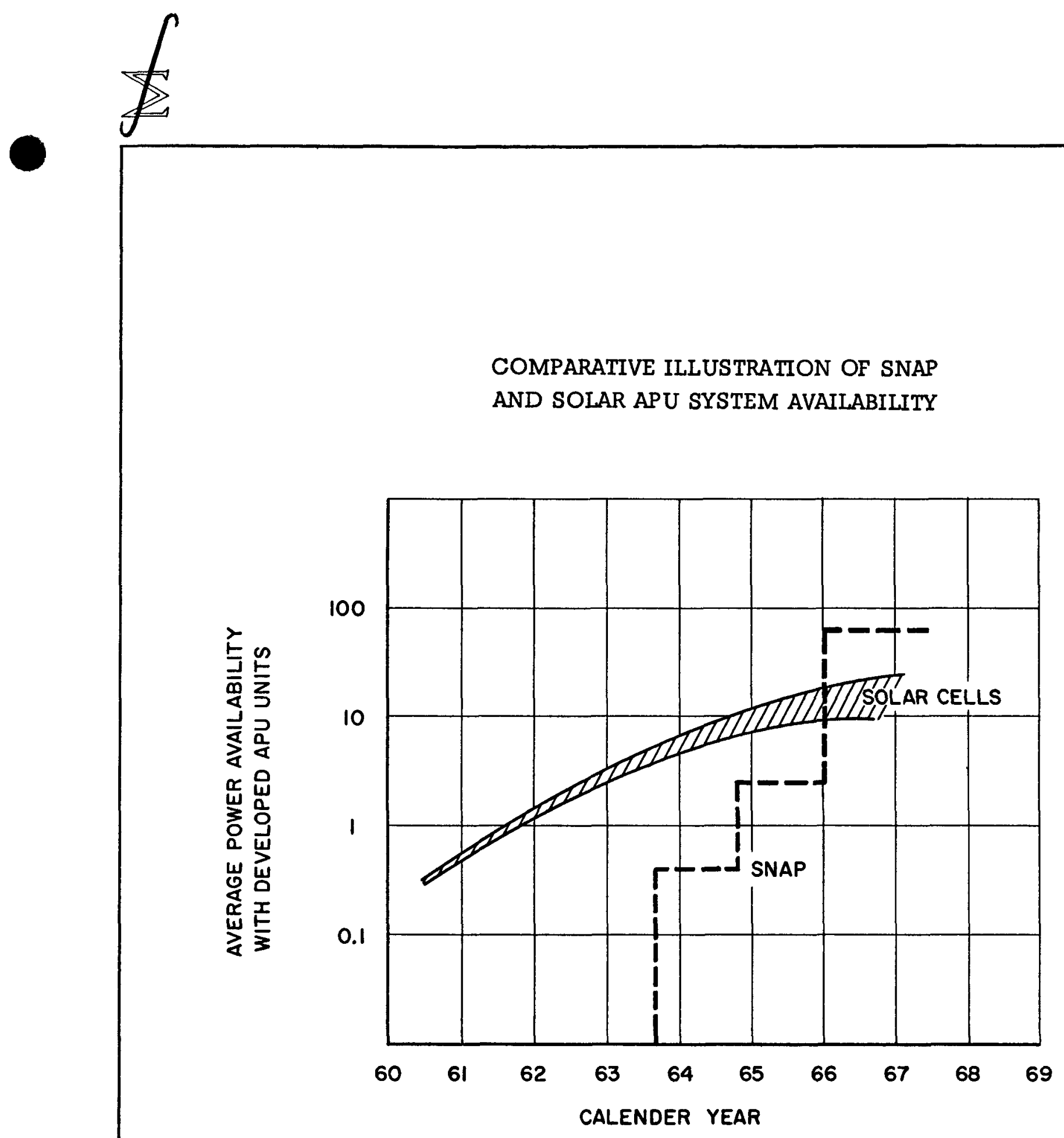

Fig. 7 


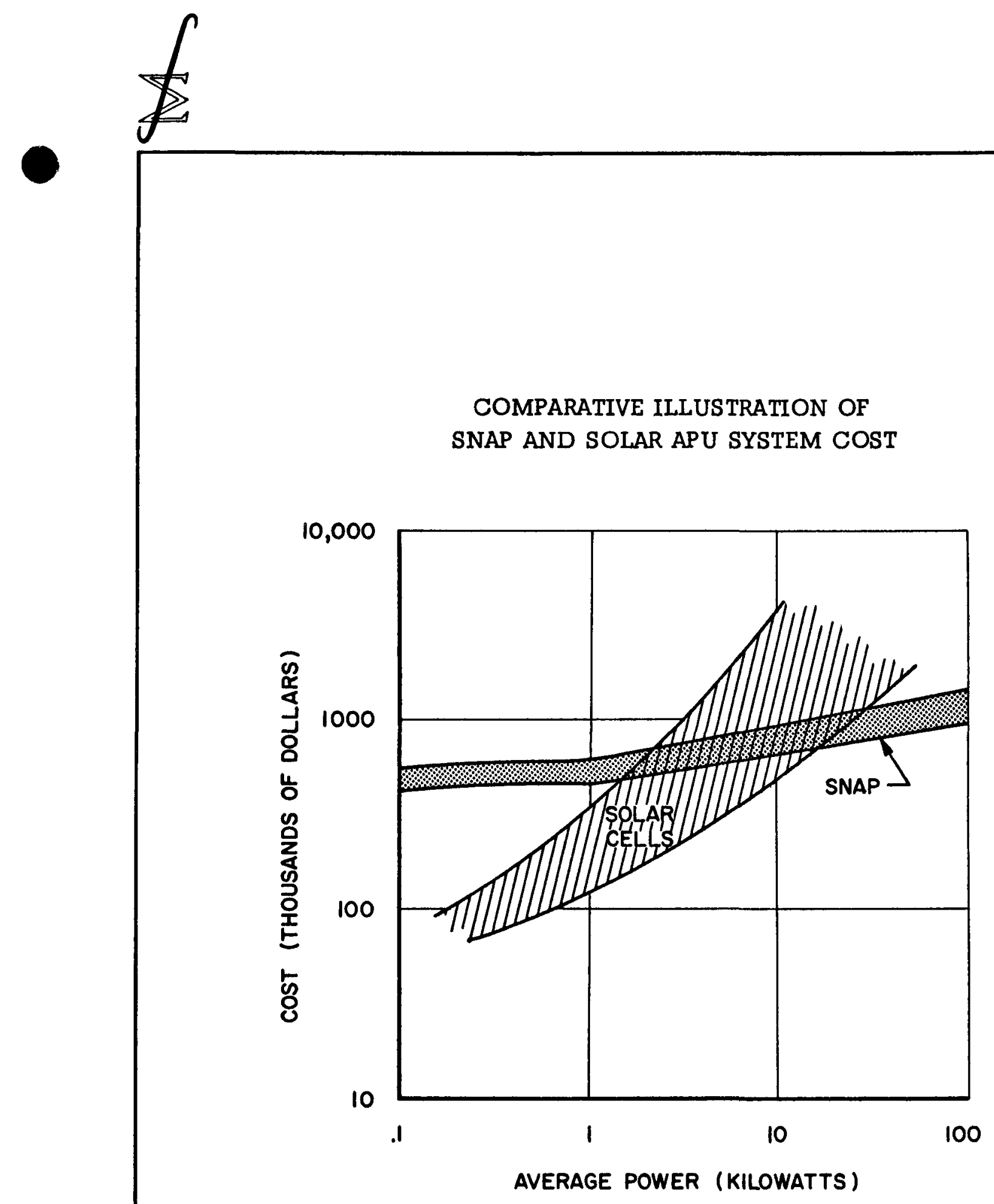

Fig. 8 
must be assignable to the APU. There is, however, a good probability that some of the shielding weight might be assignable to either system because of natural environmental effects and in this case the crossover point would be reduced.

Fig. 7 illustrates the comparison of solar and SNAP availability. At the present time systems which are designed for use prior to 1963 must of necessity be designed to utilize solar supplies. Between 1963 and 1966 solar and SNAP are competitive from an availability standpoint in the lower power regimes. There is, however, still a situation in the higher power regime where solar systems are available and SNAP units are unavailable. From 1966 on it appears likely that SNAP will be superior from an availability standpoint in the high power regime.

Fig. 8 attempts to illustrate cost in a comparative fashion. Solar supply costs will be highly dependent upon many factors but are, however, going to rise markedly as a function of power required whereas SNAP cost will rise only slowly as a function of power required. As can be seen from Fig. 8, it is expected that the crossover will lie somewhere between one and ten kilowatts . 


\section{SECTION 3 - REFERENCES}

Reference 1 - "The Practical Application of Space Nuclear

Power in the 1960's", Atomics International

paper by: J.R. Wetch, H.M. Dieckamp,

G. M. Anderson

Reference 2 - "The SNAP 2 Nuclear Space Power System.",

Atomics International paper by: J. R. Wetch,

H. M. Dieckamp, D. J. Cockeram

Reference 3 - $\quad$ "Space Nuclear Power Conversion Systems",

Atomics International paper by: C. E. Johnson,

M. G. Coombs, R. L. Hirsch 


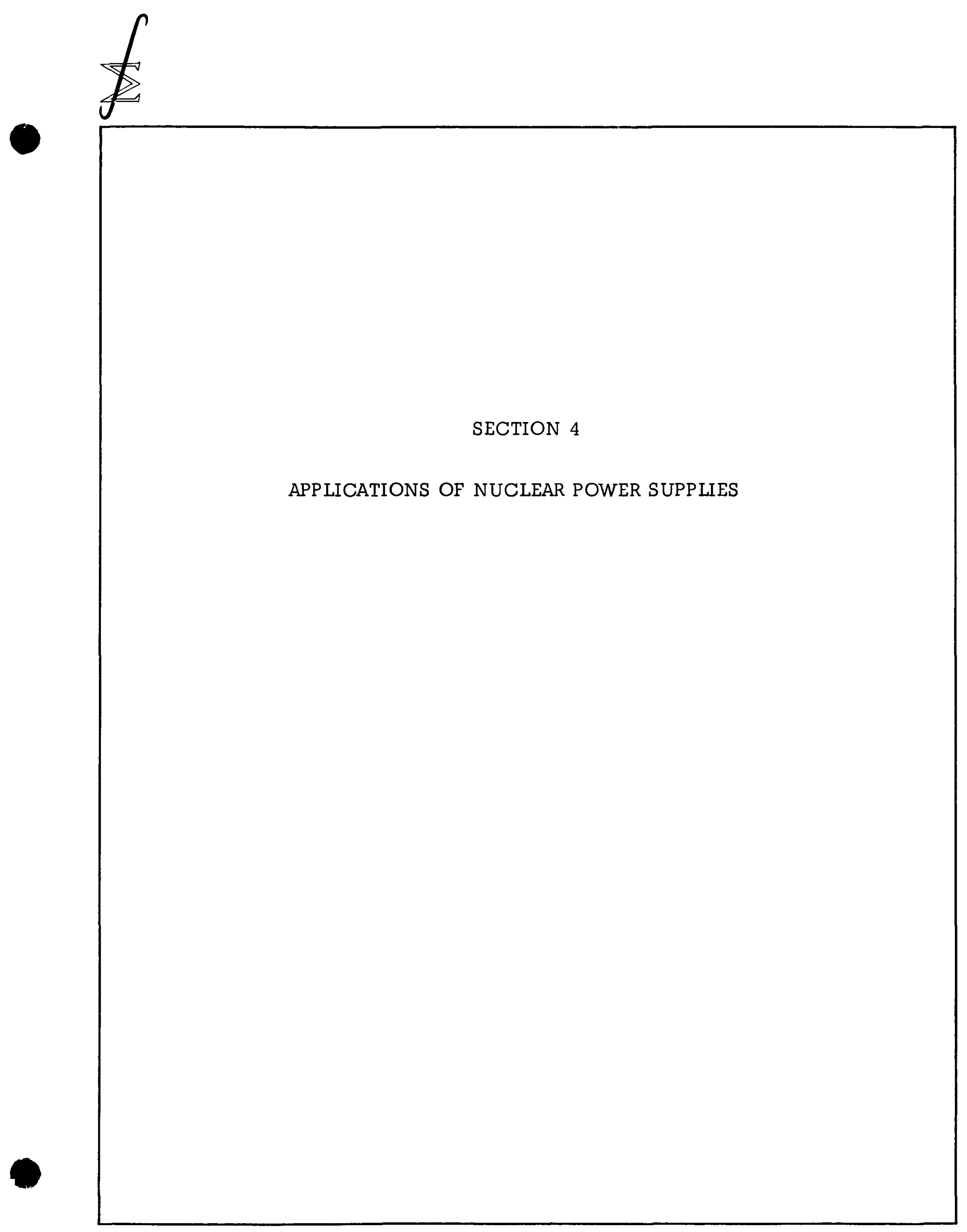

Sigma Corporation 


\section{APPLICATIONS OF NUCLEAR POWER SUPPLIES}

\subsection{Introduction}

In section 2 the U.S. space program was defined and an attempt was made to outline, insofar as possible, the probable extent of present programs and the types of future programs to be initiated. In section 3 the gross system characteristics of SNAP were presented and in particular, a comparison of SNAP and solar APU was made from a system design standpoint. It is the purpose of this section to correlate the future space program and APU and electric propulsion requirements stemming therefrom with the characteristics and capabilities of SNAP in order to define the most probable applications of SNAP.

\subsection{Applications of Current SNAP Units}

\subsubsection{Competitive Position of Present}

SNAP Units

The definition of the application of SNAPs 2, 8 and 10 is highly dependent upon their competitive position with respect to other me thods of providing electrical power. It seems fairly clear that from a competitive standpoint solar energy systems are the main concern, and in particular solar energy systems using photo-voltaic cells.

General studies tend to confirm that when weight saving is used as a measure of merit, SNAP systems do not become desirable until power requirements of 3 kilowatts or greater are specified. From the general studies on the subject the conclusion has therefore been derived that the application 
of SNAP 10 to space missions is clearly dependent upon technical factors other than consideration of energy and weight. The application of SNAP 2 to operational space systems is marginal with respect to energy and weight considerations and, therefore, decisions to utilize SNAP 2 may or may not involve energy and weight considerations as well as other technical factors. Where a requirement for 30 kilowatts of power exists, the utilization of SNAP 8 seems clearly assured.

\subsubsection{The Scientific Space Program}

Analysis of the scientific space program presented in section 2.2 in the light of estimated time scales of these flights, vehicle payload capability, APU characteristics, competitive positions, and availability of SNAP units has been performed to provide an estimate of the number of SNAP units which may be required and to identify the selected applications.

The payload capability of the Scout vehicle precludes the utilization of SNAP units for any of of the missions assigned to this vehicle. The Thor Delta firings are also removed from consideration of usage of SNAP by both payload capability and the firing schedule which occurs prior to the availability of SNAP 10 .

With the exception of two SNAP 10 development flights it also seems that payload limitations preclude the utilization of SNAP units for Thor-Agena B flights. Nova flights during this period will be vehicle development flights and are therefore not included in the summary. 
The major use of SNAP units must therefore be confined to missions for which Atlas-Agena B, AtlasCentaur and Saturn vehicles will be used and which are compatible with the availability dates of the various SNAP units.

The first part of Fig. 9 contains a summation of the number of SNAP units which migh t be utilized in the missions to be performed by these vehicles. The manned space flight category is based on the utilization of Atlas-Centaur and Satum vehicles for development flights leading to establishment of a manned space station. The earth satellite category includes the development and operation of TIROS and TRANSIT concepts as well as additional environmental study payloads having high data gathering capability. The program of lunar probes seems to be sufficiently defined at this time so as to preclude the use of SNAP units.

The category of deep space and planetary probes is predicated on the use of SNAP 2 units to provide high power for wideband communication on a few missions and the use of SNAP 8 in conjunction with EP to provide better payload capability and better communication links for probes in the latter part of the time period.

\subsubsection{The Military Program}

The military program must of necessity be evaluated on the basis of unclassified data. Considerations of the basic purpose of the programs together with logical system configurations and economic values seem to justify the 
SUMMATION OF SPACE PROGRAM APPLICATIONS

OF SNAP DEVELOPMENTS

\section{PROGRAMS}

Scientific (NASA)

Manned Space

Flight

Earth Satellites

Lunar Probes

Deep Space and

Planetary Probes

Military (Air Force)

Midas

Samos

Com-Sat

Military Exploration
2

NO. OF VEHICLES

Min. Max. Probable
SNAP TYPE AND NO.

(4) SNAP 2

(2) SNAP 8

(2) SNAP 10

(4) SNAP 2

0

0

0

$4 \quad 10$

7

(2) SNAP 2

(5) SNAP 8

$\begin{array}{lcccl}\text { Midas } & 0 & 40 & 0 & \text { SNAP } 10 \\ \text { Samos } & 0 & 40 & 0 & \text { SNAP 2 } \\ \text { Com-Sat } & 0 & 8 & 8 & \begin{array}{l}\text { (6) SNAP 2 } \\ \text { (2) SNAP 8 }\end{array} \\ \begin{array}{l}\text { Military Exploration } \\ \text { Commercial }\end{array} & 5 & 10 & 10 & \text { SNAP 8 } \\ \begin{array}{l}\text { Communications Relay } \\ \text { Com }\end{array} & 0 & 6 & 6 & \text { SNAP } 8\end{array}$

Fig. 9 
generation of a program of vehicle launchings shown in Fig. 4.

Estimates of the use of SNAP 2 in conjunction with the Samos program are predicated on the eventual development of complex payloads having several sensors in the IR, visual and radio-radar portions of the spectrum and capable of simultaneous data gathering. The operation of such a complex of payloads together with the data read-out requirement in a single vehicle will necessitate the use of a large auxiliary power supply. Whether SNAP 2 will be utilized to fulfill this requirement will depend upon SNAP performance data to be gathered from development flights and upon the relative merits of solar collectors and SNAP 2 as they appear at a later date when more operational data has been gathered.

The possible use of SNAP 10 in conjunction with the Midas payload is based on the concept that the Midas system may eventually be required to be so constructed that enemy action in the form of a nuclear burst within a reasonable sphere of influence cannot destroy the vehicle. Under this concept it seems unlikely that solar cells could be used as the auxiliary power source since they would be vulnerable to radiation and could not by virtue of their function be shielded against such radiation.

The utilization of SNAP units for the military Com-Sat program is based on the assumption that the use of SNAP 2 power will provide the basis for the simplest and most reliable system design. The two SNAP 8 launchings are considered 
to be development firings to prove out the use of EP to attain the 24-hour orbit from a low earth orbit with a communication payload. The category of military exploration is based on the assumption that a certain amount of military reconnaissance must be performed to obtain data to support military planning for future space operations in the 1970-80 period. Such reconnaissance flights will require the communication of large amounts of data from long distances and may also involve the use of Electric Propulsion.

\subsubsection{Commercial Satellite Applications}

It is likely that by the late 1960's

the capability will exist for placing a large commercial communication relay payload in a low-altitude orbit. This payload will require electrical power at about the SNAP 8 output level.

Such a system may contain electric propulsion for transition from the low-altitude to a synchronous orbit, after which the turbo-generator may be shut down and the communications power requirement satisfied by a thermoelectric conversion system of approximately 1 kilowatt output.

A very real advantage is realized by the incorporation of electric propulsion. In this manner it is possible to establish a large payload at stationary orbit altitude without requiring excessively large boosters. This factor will significantly reduce system costs, which must compete with land installations.

The number of SNAP units for this 
application are shown in Fig. 9 .

4.2.5 Chronological Development of SNAP and NPS Applications

This section presents the chronology of applications of nuclear power supplies based upon current estimates of availability of boosters and vehicles to support the foregoing scientific, military and commercial space program needs.

4.2.5.1 High-altitude Earth Satellites (1961 et seq.)

Experimental launching of earth satellites with nuclear power supplies should commence as soon as possible. The objectives of these tests are two-fold, demonstration of satisfactory design and performance in the space environment, and evaluation of the environment created by the power supply which must be tolerated by other equipment within the vehicle.

Following these tests, other earth satellites may be launched, containing payloads which utilize the multi-kilowatt electrical power available. Some of these flights may also be experimental, for example, tests of ionand plasma-propulsion systems. Others may be advanced versions of weather reconnaissance satellites equipped with recording television systems, successors to the Tiros vehicle launched in the spring of this year. Still others may be unmanned facsimiles of the manned space stations which will eventually replace them. 


\subsubsection{Point-to-Point Communi- cations Satellites (1962- 1963)}

In the sequence of evolution of communications satellites the next step beyond passive systems, such as Project Echo, will involve an active vehicle in a synchronous earth orbit, containing equipment for reception and re-transmission of voice communications.

Such a system will demand high power to minimize the complexity and costs of the ground equipment associated with it, and to provide sufficient bandwidth for a reasonable number of voice channels.

\subsubsection{Unmanned Lunar Vehicles} (1964-1966)

Early lunar probes, such as the Russian Lunik of 1959, will advance considerably man's knowledge regarding lunar topography and geography. It is assumed that a progression of instrument packages will be designed for lunar orbit and impact, and that such flights will continue through the early sixties.

Payloads designed for soft impact, for example, a small lunar observatory equipped to measure lunar and solar phenomena, may well require more power than can be supplied by solar converters of reasonable size.

Unmanned vehicles for lunar landing and return will no doubt be designed for maximum 
payload capability. Hence such vehicles may utilize combined chemical/electrical propulsion systems and will require large amounts of power.

A promising lunar application is the low-altitude reconnaissance satellite. A recording television system will be used, to store information during the times when the vehicle is in the moon's shadow. Such a satellite will be able to perform high-resolution reconnaissance and mapping of lunar topography, and surveillance of lunar bases . Power required for this mission will be high, and extended lifetime of operation is desirable, both requirements which can be satisfied by nuclear power supplies .

\subsubsection{Manned Satellite Stations}

This mission requires

the capability for sustaining operations of several personnel in a more-or-less permanent orbit. Most authorities consider that orbit altitudes in the range of 300 to 500 miles will be high enough to provide orbit lifetimes in the order of several years, although at the lower limit small propulsion increments may be required periodically to compensate for decay due to atmospheric drag. Operations at altitudes much higher than 500 miles will subject the station to intolerably high solar-radiation levels, and will require prohibitive amounts of shielding to protect personnel within the station.

Current literature con- 
tains many estimates of the power required to sustain life during extended time periods of operation in space. These estimates vary from 1 kilowatt per man as a minimum estimate, up to about 5 kilowatts per man. Since it would be clearly unwise to permit such a complex and useful vehicle to become power-limited, it would seem wise to accept as a criterion for design something like 8 kilowatts per man. This will provide a satisfactory margin for operation of all equipment as well as direct life-support which will be installed in the vehicle.

Assuming that this vehicle will be manned by at least four persons, then about 30 kilowatts of auxiliary power will be required.

4.2.5.5 Manned Lunar Missions (1967-1970)

The first manned lunar flights will be limited to simple circumlunar trajectories. For reasons of safety, these flights will probably be initiated from, and terminate at the manned satellite stations discussed above. Although it is certainly conceivable that cis-lunar flights direct from Earth and return may be made, transit times will in any case be at least ten days or more. It is not considered likely that lifesupport could be reasonably sustained for such periods by energystorage systems. Hence, solar or reactor power supplies will be required.

Low orbit reconnaissance, soft landings and return, and the establishment of manned lunar 
stations will follow the initial manned lunar probes. Each of these systems will be extremely complex, each will require large amounts of auxiliary power for life-support and for the operation of system equipment .

\subsubsection{World-Wide Communica- \\ tions Satellite Network}

The application of nuclear power sources for point-to-point communications was discussed in section 4.2 .5.2 above. This mission may be extended in scope to the provision of several satellites in a synchronous orbit for worldwide communications. The economies of this system are particularly attractive, because of the high cost of installation and ma intenance of land networks.

To be commercially feas ible, the satellite system must show superiority over land-lines and micro-wave relay networks both in performance characteristics and in specific operating costs (cost per channel per unit time). It is estimated that direct costs for the satellite system would exceed those of equivalent direct systems by about fifty percent at present. In the immediate future, however, current development programs in space communications equipment will bear fruit, and it is anticipated that within the next five years satellite systems will surpass land networks as an efficient, economical medium for civil communications on a world-wide basis .

Of course, all facets must 
be examined, and evaluated in the light of their respective problems. These facets are first the vehicle-borne equipment, second, the ground installations, third, the ground distribution network and finally, the operational procedures which must be developed to prevent saturation and break-down of the system. No problems in these areas appear insurmountable.

\subsection{5 Unmanned Interplanetary \\ Missions (1967-1972) \\ These missions, essen-}

tially the continuation of the international programs of deep space probes, will be considered briefly, with emphasis upon more sophisticated payloads and on the probable requirement for electric propulsion. It will be noted that there is unique need for nuclear power supplies with this propulsion medium.

The early years of this decade will yield many more answers to our questions on space environment and our near planetary neighbors. It will remain, however, for much more advanced payloads to evaluate fully the phenomena first discovered by the initial probes. This second generation of interplanetary vehicles will therefore require more complex and more power-demanding equipment than their predecessors.

Electrical power requirements for propulsion are impressive, and they are not likely to be satisfied by solar energy conversion systems. Direct application of solar radiation for propulsion, solar "sailing", is certainly of interest, at least in combination with other types of propulsion. 
Nevertheless, there is a clear role for nuclear power supplies to meet the large, continuous demand for propulsive energy exhibited by interplanetary vehicles.

4.2.5.8 Manned Interplanetary

Missions (1972 et seq.)

This mission is, of course,

man's ultimate objective in space. To meet this objective will require integration of many technologies, and the contributions of many special fields of human knowledge. It is, therefore, not at all presumptuous to discuss the role of any single item of equipment, for on the operation of each will depend the successful flight of the vehicle and the survival of its crew.

Flight times of a manned vehicle, from an earth-orbiting space station to the vicinity or to the surface of even our nearest planetary neighbor will be measured in months and years, with corresponding times for the return flight . Cumulative power requirements for life-support systems, instrumentation and communications, and propulsion and vehicle control of 150 to 200 kilowatts are expected, and may eventually grow to the megawatt regime.

\section{Nuclear power supplies}

are now being developed which may be manifolded to provide these initial amounts of power. There is no doubt that their performance and reliability will have been amply demonstrated in this decade, and that they will be fully utilized in manned interplanetary craft. 


\subsection{Applications for Future Nuclear-Power Supplies}

It is not consistent with past history to expect that specific auxiliary power requirements will diminish as larger payload capabilities are reached. The rate of increase in these power requirements is not clear, but it is likely that these requirements will continue at least at the present ratio and will probably increase by as much as $30 \%$. Life support systems of any reasonable operational duration, that is, in excess of a few days, will impose a demand for power in the order of several kilowatts per man on top of the conventional power requirements for guidance and control, instrumentation and communications. The development of electric propulsion systems will result in similar large incremental increases in the demand for electric power.

This trend is illustrated in Fig. 11, as a function of payload weight and also as a function of time, based upon the growth in payload capabilities shown in Fig. 10. This figure also must be accepted as a reasonable estimate only, since it is virtually impossible to determine at this point in time the actual characteristics of payloads which are not yet designed. It is presented as a sort of lower limit estimate of the demands of the increasingly more complex payload systems which will emerge.

In part, future applications for nuclear power supplies may require only extension of design limits on then existent units, alterations of configurations, combinations of conversion units or other similar excursions to extend development capability into operational applications. Other future appli- 


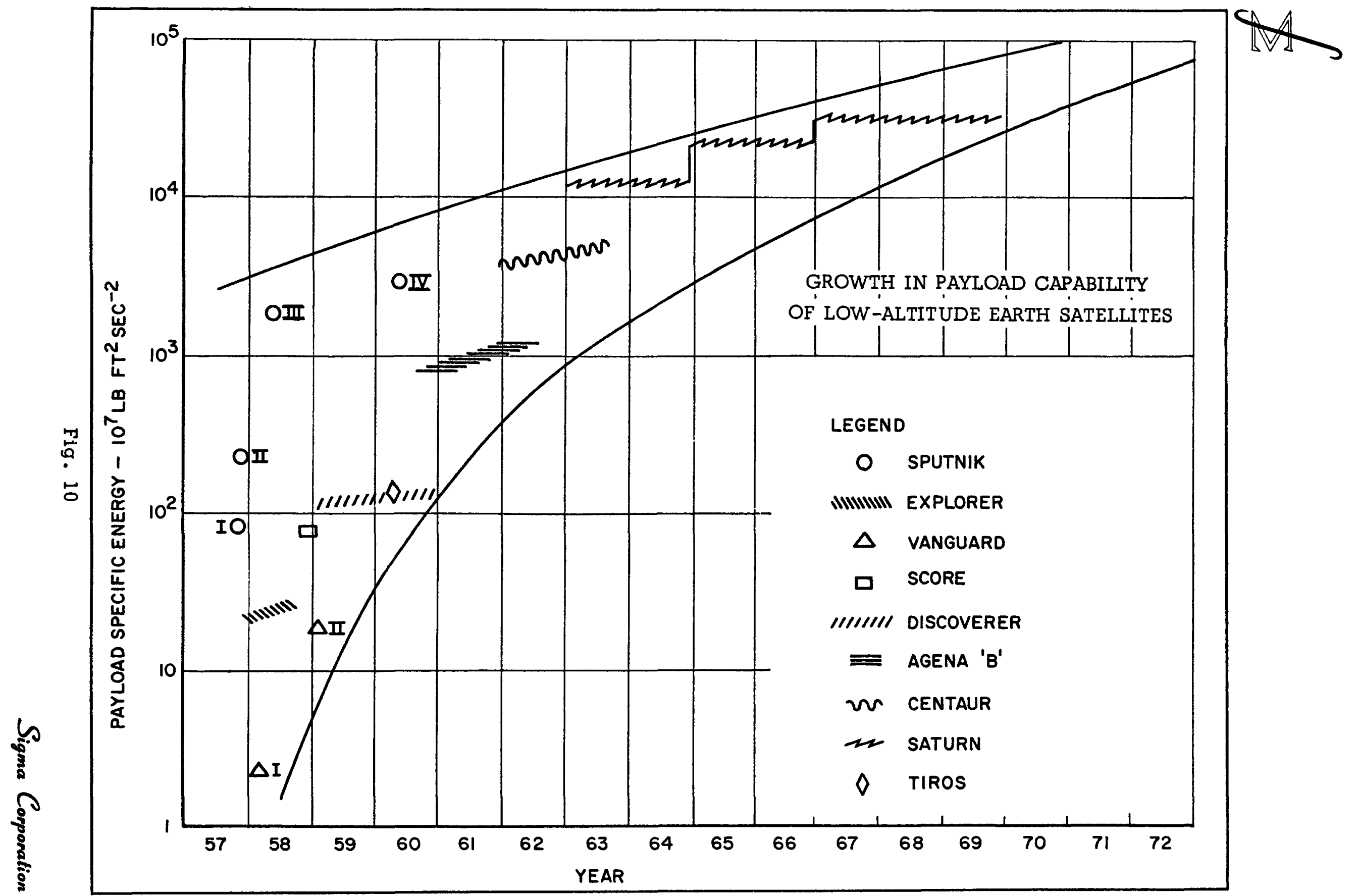


cations will exceed present technological limits, or will be sufficiently major departures from existing units to warrant or require major development effort. New integrated or direct conversion units will be needed for electric propulsion at power levels of 100-300 kilowatts with conceivable later requirements as high as 3 megawatts. Fixed base power requirements in space will require large capacity nuclear power units commencing with SNAP 8 reactors for the initial earth satellite station and growing to 3 megawatt levels with maturation of the lunar base.

Integrating the expected demands for power with the development schedules for SNAPs 2,8 and 10 provides some insight into the capabilities which systems utilizing these units will possess. In Fig. 12 are plotted the demands for nonpropulsive power resulting from the preceding analysis and a tentative curve for electric propulsion power. The availability of SNAP units is superimposed on this plot to assist in the determination of the applications .

Requirements for non-propulsive power, as mentioned above, are based on predicted growth of payload capabilities. Propulsive power requirements are determined by the loci of thrust acceleration required for several types of missions. The first of these, experimentation with electric propulsion units, may be satisfied by low-level power systems, providing thrust accelerations in the order of $10^{-5} g_{0}$. These systems will be used for propulsion development in the time period 1964-1965. The intermediate level of propulsion requirements, $10^{-4} \mathrm{~g}_{\mathrm{O}^{\prime}}$, will permit 
operations from low-altitude earth orbit to stationary orbits and cis-lunar flights with payloads in the order of 3,000 pounds. This requirements will appear in the 1965-1966 period. The upper level of propulsion power requirements, $10^{-3} g_{0}$, will provide the capability for manned interplanetary operations, such as cis-Martian and Cis-Venusian flights of approxima tely two months duration. The following conclusions may be drawn from Fig. 12:

(1) SNAP 10 possesses marginal capability for new systems because of its late operational availability date. By 1963, payload power requirements will approach 1.5 to 2.0 kilowatts. Development of this unit is encouraged, however, because of its potential application to operational systems growing out of present development program. These systems will not possess payload capabilities equal to those available from the new systems of 1963, but they are likely to be evolved to the point where the long operational lifetime and high reliability of the thermo-electric power conversion system will become attractive and competitive with solar-conversion systems .

(2) SNAP 2 possesses clear capabilities for satisfying the non-propulsive power demands of the most advanced payloads of the 1964 operational systems. It is perhaps marginally useful also for operations in conjunction with the development of electric propulsion systems, if one is willing to sacrifice long lifetime by operating the unit at two or three times its designed output. 
(3) SNAP 8 represents the first unit which has capabilities for exceeding, but a substantial margin, the probable demand for non-propulsive power when it becomes operationally available in 1966. This unit also possesses capabilities for electric propulsion development at its designed output level, and may well be capable of providing propulsive power for transition trajectories from low-altitude earth orbit to stationary orbits, if operated about its rated output level.

(4) The 300 kilowatt unit development program should be accelerated to become operational in mid-1967. At this point in time, it will be capable of providing propulsive power for operational manned cis-lunar missions as well as for uninhabited experimental tests of manned interplanetary vehicles.

(5) Consideration should be given at an early date to the initiation of a development program for a 3,000 kilowatt system. This conclusion can be intuitively supported at this time, although a far more thorough analysis must be undertaken to determine the validity of this requirement and its target operational date. Such a unit would provide the capability for interplanetary craft occupied by several personnel, and in fact, may well represent the threshold value below which the ultimate objective of planetary exploration cannot be achieved. 




Sigma Corporation 


\section{DESIGN INTEGRATION}

\subsection{Configuration and Installation}

Integration of SNAP systems into a vehicle permits three general installation concepts. The first concept consists of disposition of all SNAP components within the basic envelop of the vehicle, as space and balance limitation allows. This approach is similar to aircraft design standards, and in most cases would not be satisfactory for any major subsystem, particularly for a nuclear power supply. The second concept is the mixed approach, with all elements but the reactor packaged in the basic envelope of the vehicle, and the reactor remotely installed or extensible. This approach seems logical and feasible, except for the mechanical complications associated with long flexible lines, slip joints and folding or telescoping members, all hermetically sealed. The third concept, favored for Agena B applications, is the "nose cone" installation. Here the entire SNAP system is a detachable, pre-assembled and pre-tested unit, requiring only mechanical and electrical connection between itself and the vehicle.

If power requirements are small, for example in the nominal SNAP 10 range, the power supply may probably be installed anywhere in or on the vehicle, and the only limit on choice of configuration involves the amount of shield necessary to protect the basic vehicle subsystems and payload. If power in the amount available from SNAP 2, or in greater amounts, is required, then it is reasonably certain that the power supply will be a determining 
factor in the ultimate design. This does not necessarily mean that the entire vehicle will have to be designed around the power supply, but merely that some priorities must be assigned to its requirements much as the extreme aft end of a vehicle is reserved for the installation of a rocket engine.

Performance and efficiency currently being achieved allow some rule-of-thumb estimates to be made as to power system specific weight, radiator specific area and shielding required as a function of type of payload to be carried. It is not recommended that current values for these parameters be used as design criteria for vehicle systems to be developed in the future, simply because they are quite sensitive to improvements in efficiency of individual components of the power supply.

Power supply components which most influence vehicle design are the reactor and the radiator. Optimum reactor location dictates maximum feasible separation distances, to minimize the cone angle to be shielded. This gain diminishes, however, in terms of separation length, when the included cone angle has been reduced to about $30^{\circ}$. Total cone angles of much less than $20^{\circ}$ are likely to result in structural problems at the mating plane which begin to offset further advantages in shield reduction because of the high fineness ratio of the combined body. Similarly, surface area of the cone, if used as a radiator, is not optimized.

The radiator itself presents some design integration problems, since it is not adaptable as a load-carrying surface. 
Many configuration choices are available however, although the classic concept of "simplest equals best" may apply. The radiator may be flat, or it may be any simple curve, for example cylindrical or conical. Flat surfaces are not likely to be feasible, because they cannot be stored conveniently within the envelope of the vehicle, or developed easily from its external contour. Cylindrical surfaces seem to be nearly optimum, from the specific weight standpoint, and they may be fixed, extendible as a unit, or segmented. The segmented cylindrical configuration may be extended about one end or about a longitudinal edge. In the latter case, it is conceivable that they might serve as part of an antenna system, fed by a dipole array on the cool side, if thermal noise is not excessive. A conical configuration, for the Agena B installation, is discussed briefly in section 6 .

Remaining elements of the nuclear power supply, such as the heat-exchanger, converter and controls are reasonably small and light, and from the configuration point-of-view, do not introduce significant integration problems.

\subsection{Environmental Effects}

The primary environment created by the SNAP systems is that of radiation from the reactor. Virtually every component and subsystem of the vehicle requires shielding from this radiation, and shielding at the source is clearly superior to local shielding, except for specific elements which may be more sensitive to this environment than the majority of the others. This exception, which applies to some of the payloads discussed below, 
requires a combination of source and local shielding, to result in a minimum total system weight. The secondary environment is thermal, involving radiation and conductive heat transfer from the power supply. Of the two processes, radiation represents by far the greater source of heating, and conduction will only affect equipment and structure adjacent to the radiator and boiler.

Shielding against radiation from the reactor can provide, depending upon the configuration and weight allowed, adequate protection to typical electronic components for systems of one year's lifetime, and the specific weight (lb./kw) remains competitive with other sources of energy.

Protection against the thermal environment should not require excessive design measures. If the radiator elements are extended, then no significant thermal radiation will be felt by the vehicle. If it is fixed, then internal reflective surfaces should suffice to prevent heating of vehicle equipment and payload.

The mission analyses contained in preceding sections of this report identify several applications of SNAP systems in scientific, commercial and military space programs. Payload equipment can be inferred from these applications, and the sensitivity of such equipment to the environment created by SNAP systems can be examined. Each mission will likely involve one or more of the following basic categories of payload: scientific instrumentation, communications equipment, military reconnaissance systems, electronic and electromechanical systems, and biological 
support systems.

SNAP systems should find application on deepspace and planetary probes, because of the long communication distances involved, and the desirability of having sufficient transmission power (band-width) to avoid excessively slow-time readout of instrumentation data. Unfortunately, the purpose of the instrumentation for such mission demands that the environment which it is to measure be uncontaminated by the vehicle which bears it. Particularly, cosmic radiation in space and planetary atmosphere detection, subjects of importance and much interest, are measurements which are easily distorted by the radiation emitted from the reactor. A high degree of local shielding, small aperture, and perhaps very remote location will probably be required. Small instrumentation packages, similar to radiosonde devices, might be discharged from the vehicle on command, and transmit their data to the parent vehicle for relay to earth. In this manner, virtually uncontaminated measurements could be assured, for such a package even of minimal size should have a transmission range of a few hundred miles. Also by this technique direct measurements of planetary gravity fields could be obtained. Other scientific instrumentation sensitive to the radiation environment might be infra-red and TV scanning devices, used to determine the presence of organic life. Both of these devices are reasonably tolerant to radiation, but their information output would be significantly degraded by noise even though their basic functions are not destroyed. 
Communications equipment presents no major problem areas at the levels of exposure easily maintained at reasonable shield weights, and signal-noise ratio is much more improved by the high power available than it is degraded by radiation effects.

\section{Military reconnaissance systems will consist of} electron, infra-red and visual sensing devices similar to the payloads of Agena vehicles. It is possible that combinations of two or more may be installed in one vehicle, thereby reducing the ambiguity and increasing the value of the data received. Visual data systems are more likely to involve television and electromagnetic tape storage than optical equipment and photographic film, for reasons of comparative performance. If this is so, then it is hard to define any basic incompatibility of the payload with SNAP power supplies.

Functional electronic and electromechanical equipment within the vehicle is also tolerant of the reactor environment when a reasonable source shield is provided. This type of equipment is more susceptible to gradual deterioration in performance than it is likely to suffer sudden impediments to its operation or catastrophic failure. Continuing experiments to evaluate radiation effect, and materials and design improvements, should yield components capable of at least one order of magnitude higher radiation tolerance by the time SNAP systems are available for installation in future sophisticated vehicle systems.

Of all the problems associated with life support 
(plant or animal) in space, radiation from the reactor is certainly not the greatest, if it is contemplated that the system operate at or through the Van Allen radiation belts or into cislunar or interplanetary space. While it is true that shielding requirements for man exceed those of electronic components by a factor of ten, the specific weight of the nuclear power supply system diminishes rapidly as the power level increases. Thus the system tends to optimize itself, because of the high power required for life support. 




Sigma Corporation 


\subsection{Agena B}

The following paragraphs contain a brief summary

of factors to be considered in the integration of SNAP 2 and SNAP 10 with Agena B vehicles. This subject is considered from two aspects, namely performance and design. Since no direct liaison was performed with LMSD, and since the study was performed on an unclassified basis, detailed and definitive data are not provided.

It is necessary first to list all items of equipment which consume electrical power, and to define their input voltage and frequency, regulation requirements, nominal and peak power requirements and duty cycle. This list will determine the resulting total demand and variations which determine the output level of the SNAP system and the requirements for output control. Much of these data are already available to AI, but it is assumed that no decision has been made regarding the incorporation or selection of an Agena-type payload for these flights. It is recommended that some such payload, or simulated payload be included, though on a priority subject to that of the SNAP system itself.

Operation of the power supply may introduce certain problems in vehicle altitude control. The magnitudes of the moments of inertia and angular momenta computed for SNAP 2 appear likely to introduce only negligible disturbances during steady-state operation, although start-up accelerations of the combined rotating unit will probably require that the vehicle 
stabilization system be active during this period. This stabilization is desirable, in any case, because successful start-up is a primary objective of the tests, and irrelevant environmental effects of random or unstabilized vehicle altitude will want to be avoided.

Control of the power supply will probably be demonstrated first at a quasi-steady-state output, perhaps for several orbit revolutions. It may then be desirable to cycle the power load to demonstrate adequate system response. The complete series of test conditions should include all nominal and offdesign ranges determined by analysis of the entire vehicle equipment array, and the experiment should be programmed to verify the results obtained from qualification tests conducted prior to flight.

All of these tests will contribute to the total instrumentation and data requirements, both in number of instruments and channels as well as in integrated telemeter coverage from the ground stations. It will be necessary, of course, to examine the entire active trajectory, so that test operations requiring realtime data or control can be scheduled to coincide with phases of orbit when read-out and command capability can be assured.

Design integration involves the physical and environmental factors associated with the actual installation of the SNAP systems in an Agena vehicle. Of the two basic configurations considered, the submerged ("modular") and separable ("nose cone"), the latter appears to have been at least tentatively selected and the most promising. 
The merits of this configuration which led to its selection need not be repeated here, but the design problems associated with it can be reviewed briefly. First, the disposition of components, with heavy elements located in each longitudinal extremity, results in the highest possible moment of inertia about the pitch and yaw axes. These moments must be computed to determine the bending moments at the mating plane and the effects on gas requirements for vehicle stabilization. Also, the gross weight of the complete installation exceeds the nominal gross payload of the Agena vehicles by several hundred pounds, and will affect the launch trajectory as well as the structural integrity of the vehicle.

Second, the thermal environment created by the radiator and internal heat losses may require supplementary temperature control measures for other vehicle equipment and payload. In the SNAP 2 system, internal heat losses throughout the cycle, particularly in the boiler and piping and back radiation from the radiator, may generate a heat flux of 2 or $3 \mathrm{kw}$ across the mating plane. Presumably a reflective diaphram will be installed to minimize heat transfer by radiation. An additional small fraction of the 40 kilowatt thermal rejection may be transferred by conduction to the vehicle structure. This environment is not considered to be intolerable, but it is likely that a significant percentage of the 45 to 50 kilowatt thermal output will be felt by the vehicle unless this factor is taken into account in the design integration. The thermal environment created by the SNAP 10 in- 
stallation is probably much less severe, due to the physical arrangement of its components, and operation of the reactor at a lower thermal output level.

Third, the radiation environment itself should create only limited problems if it can be assumed that objectives of the experimental flight tests will not limit the weight allowance for shielding. Even so, it would be of more than passing interest to attempt installation of some sort of reconnaissance payload, to evaluate the performance of electronic components when exposed to this environment.

In summary, it may be said that the installations of a SNAP 2 or SNAP 10 system will present the designer with neither the simplest nor the most difficult integration problems. These systems are certainly as tolerant of the launch and ascent trajectory environment as any of the basic vehicle equipment. Therefore, no design compromises will be required for protecting the SNAP installations, other than the provision, perhaps, of a frangible or jettisonable non-metallic cover over the radiator and reactor. Structural and mechanical integration should be no more difficult for these systems than for any other payload of equivalent weight and dimensions. If it can also be assumed, and it is certainly recommended here, that the reactor is not brought up to operating level before launch, then only a minimum amount of effort will be required to integrate this equipment with the pad and blockhouse monitor and control system. 


\subsection{Other Vehicles}

Forecasts of the scope of U.S. Scientific, civil and military space programs are based upon the design capability of Atlas-Centaur and Saturn booster systems as launching devices for large satellite and space vehicles. Booster performance goals are fairly well-defined, but no such definition is available concerning the actual final-stage vehicles themselves.

Integration of SNAP systems with these vehicles was discussed categorically in section 5 of this report. Little more can be added until vehicle details become known. Nevertheless, it is by no means premature to begin liaison with the agencies responsible for these developments, for an optimum system design should include integration of power supply requirements with those of other major subsystems. Furthermore, if actual power requirements demand, for example, operation of SNAP 2 at 4 or 5 kilowatts, then on a specific weight basis clear superiority over competing (solar) energy sources may be shown. Feasibility of such modes of operation needs be thoroughly examined as control, reliability and life duration are affected.

It can be postulated that integration becomes easier, and application more universal, as a direct function of the ability of AI to provide a compact module of reactor, boiler and controls, and a "rubberized" radiator design which can be matched easily to the actual power requirement and vehicle configuration. 


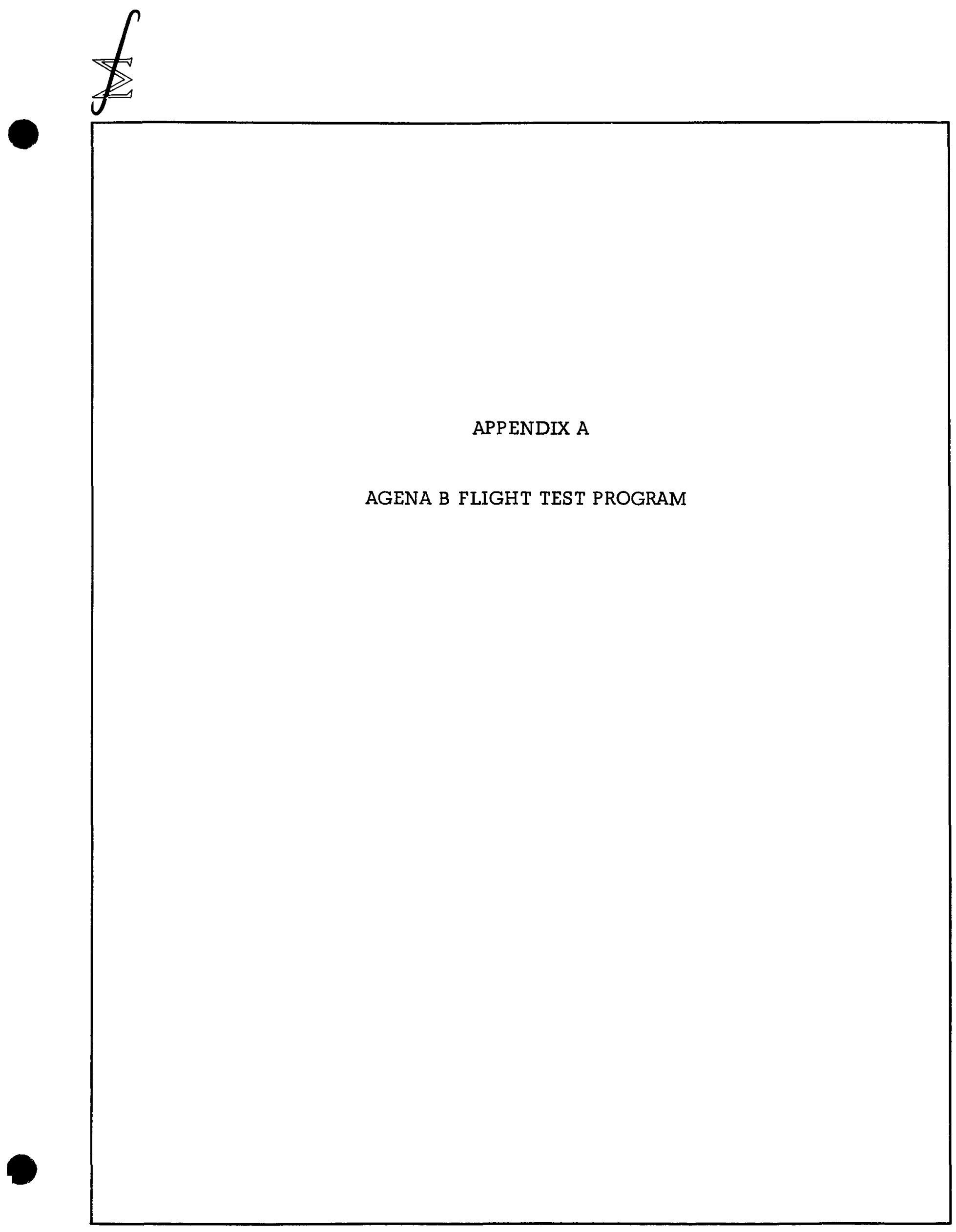

Sigma Corporation 


\section{APPENDIX A \\ AGENA B FLIGHT TEST PROGRAM}

\section{Introduction}

This appendix contains an outline of the contents of a test plan for the flight programs of SNAP 2 and SNAP 10 in Agena B vehicles.

The outline lists by topic heading those requirements and procedures necessary to the successful conduct of the tests. Some of the contents will be furnished entirely by AI; some will be prepared by LMSD, based upon requirements generated by AI; and some also must be prepared by AI to satisfy requirements of LMSD.

Definitization of the contents of this plan obviously requires access to more information than can be made available on an unclassified basis. It also requires continuing liaison between the several contractors involved in the program.

\section{Flight Test Plan Outline}

1

Test Objectives

$1.1 \quad$ Primary

1.2 Secondary

2 Configuration
2.1
SNAP
2.2
Vehicle
2.3
Payload 


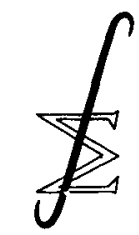

3

Trajectory

3. 1 Launch

$3.2 \quad$ Orbit

4 Data Requirements

4.1 Tracking

4.2 Flight Instrumentation

4.2.1 Pre-launch and Ascent

4.2.2 Orbit

5

Pre-launch Operations

5.1 Hangar

$5.2 \quad$ Pad

5.2.1 SNAP

5.2.2 Launch Integration

$6 \quad$ Post-launch Operations

6.1 Command and Control

6.2 SNAP Start-up

6.3 SNAP Operations

$7 \quad$ Support Requirements

7.1 Contractor Facilities and Equipment

$7.2 \quad$ Ground Support Equipment

7.3 Contractor Personnel

7.4 Government Furnished Equipment

$7.5 \quad$ Range Support

$8 \quad$ Range Safety

8.1 Test and Checkout

8.2 Pad Operations 


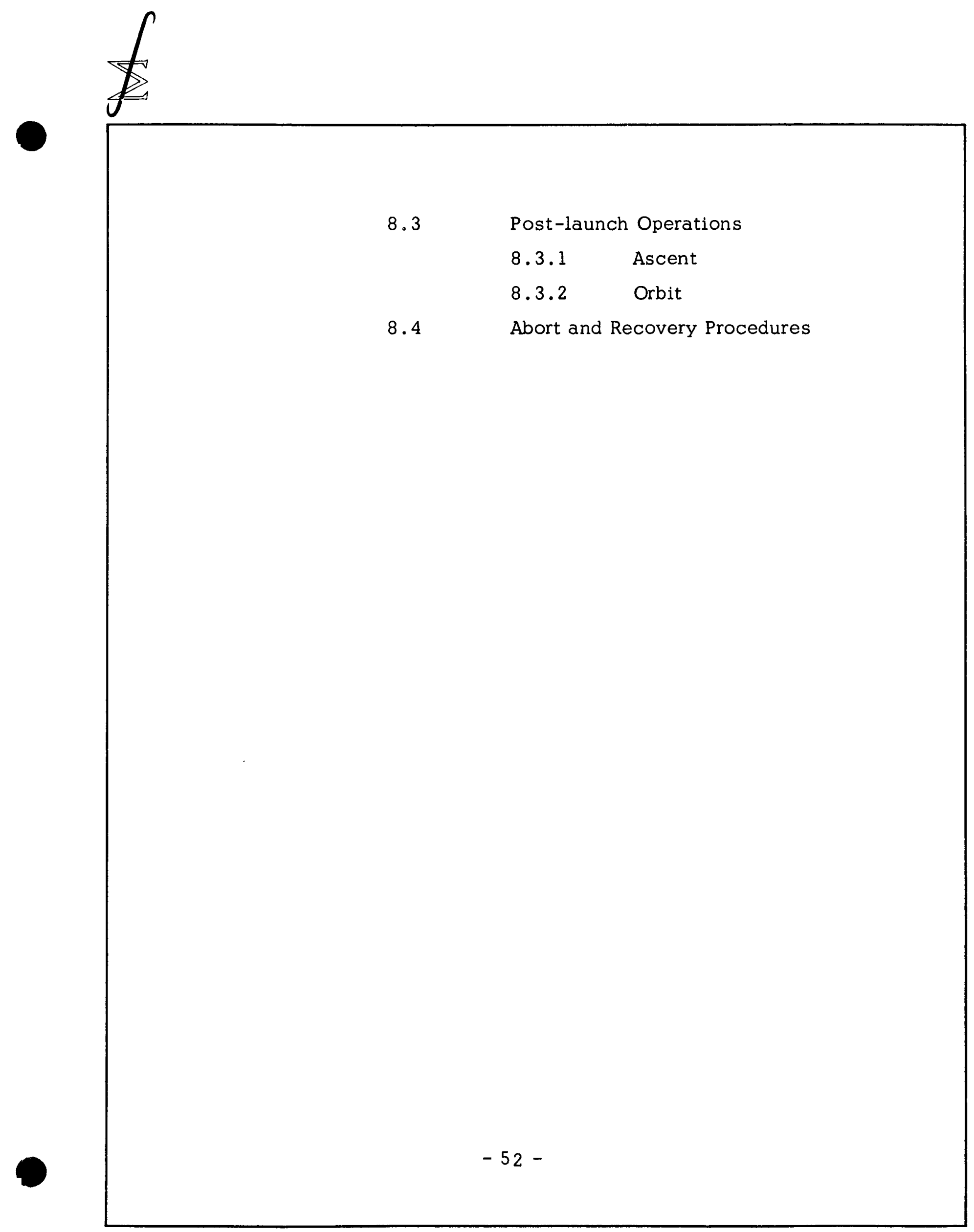




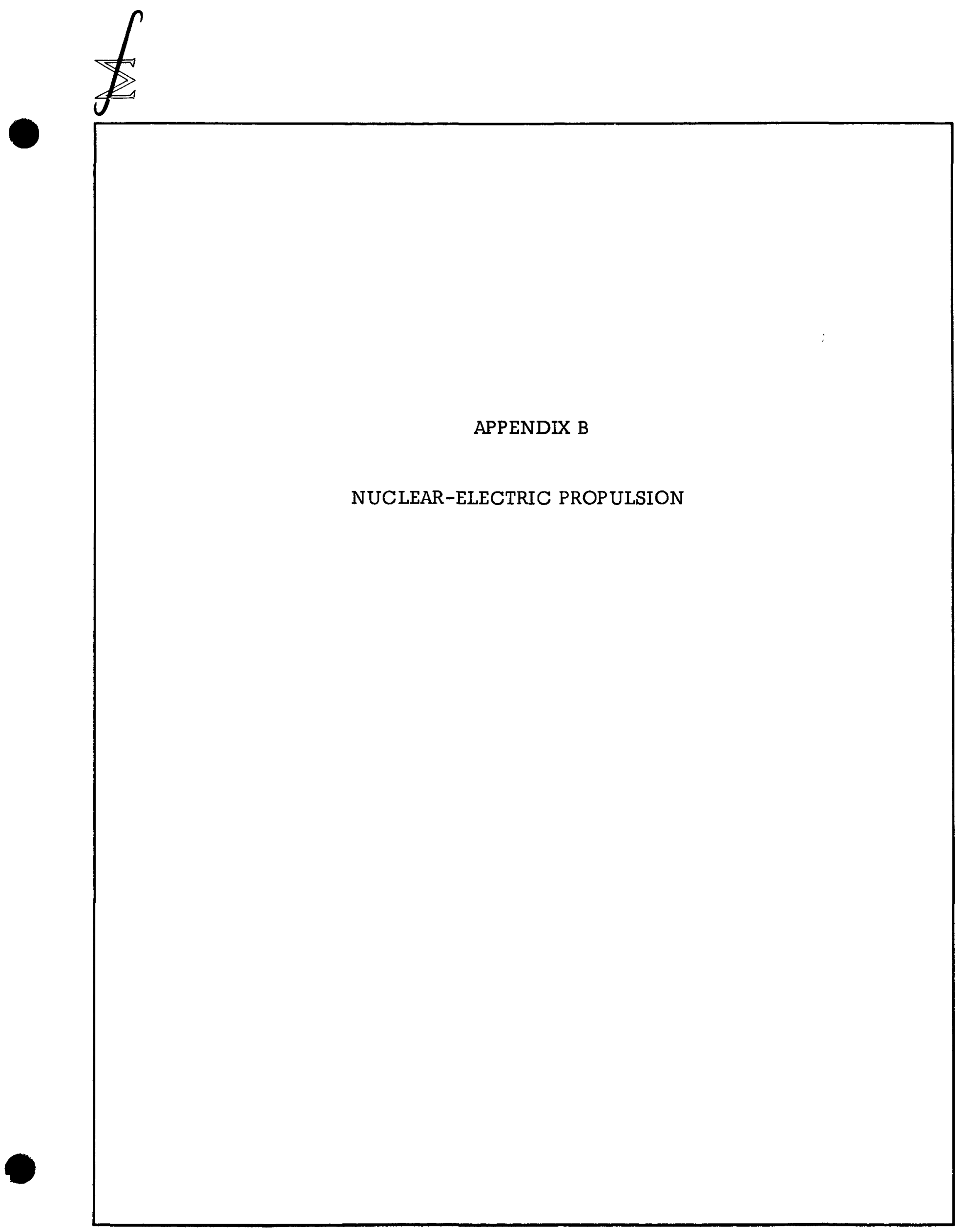

Sigma Corporation 


\section{APPENDIX B \\ NUCLEAR-ELECTRIC PROPULSION}

The major components of a nuclear-electric propulsion system are (1) the nuclear reactor, which generates thermal energy, (2) the thermo-elctric conversion stage, which converts the thermal energy into electrical energy, and (3) the propulsion stage, which converts the electrical energy into directed thermal energy in a manner which produces propulsion. Stated in this manner it is clear that the arrangement of stages could well miss the second step, particularly since it is at present so inefficient. However, if the second step is eliminated, the system becomes a nuclear propulsion system rather than a nuclear-electric propulsion system. Consequently, by definition then, the thermoelectric conversion stage is part of the system and attention should be given to increasing its efficiency.

It is not being unnecessarily arbitrary in including the conversion stage because nuclear-electric systems do have some advantages over nuclear propulsion systems. They are far more amenable to control than any of the nuclear-propulsion schemes so far proposed, and they offer hope of being useful in practical systems much earlier than the nuclear-propulsion systems. While the apparent thrust-weight ratio of the nuclear-electric systems is marginal when considered in terms of planned missions and considerations of payload versus thrust, not much of an improvement 
is required to make them quite satisfactory. Furthermore, they offer higher specific impulses and present fewer engineering and design problems. One disadvantage is, of course, the requirement for a booster to get the system into space before it can function. However, Fig. B-1 shows that most of the other nuclear schemes are marginal for use as boosters, not even considering the associated radioactivity which would probably prevent the use of even those systems which, could function as boosters for missions from earth. (The figure is adapted from a plot in Astronautics, vol.4, no. 10, p.25, Oct.1959, and a description of the various nuclear systems can be found there.)

One type of conversion system presently being developed for nuclear power units are the mercury turbo-generator with conversion efficiency of around $10 \%$ and a solid-state system with somewhat less efficiency. There are, however, many other conversion systems under development or research, such as vacuum or cesium diodes, and magnetohydrodynamic systems. The solid state devices appear to be limited in the maximum temperatures at which they can operate and not deteriorate. The vacuum or cesium diodes require temperatures of nearly $1000^{\circ} \mathrm{C}$. before they become competitively efficient with turbo-generators, as indicated in Fig. B-2. The magnetohydrodynamic systems will apparently be constrained to use in very large systems and will apparently require higher-temperature sources than the diodes. As a consequence, at the present time the diode system appears to offer the most hope and advantage for near-future systems. 


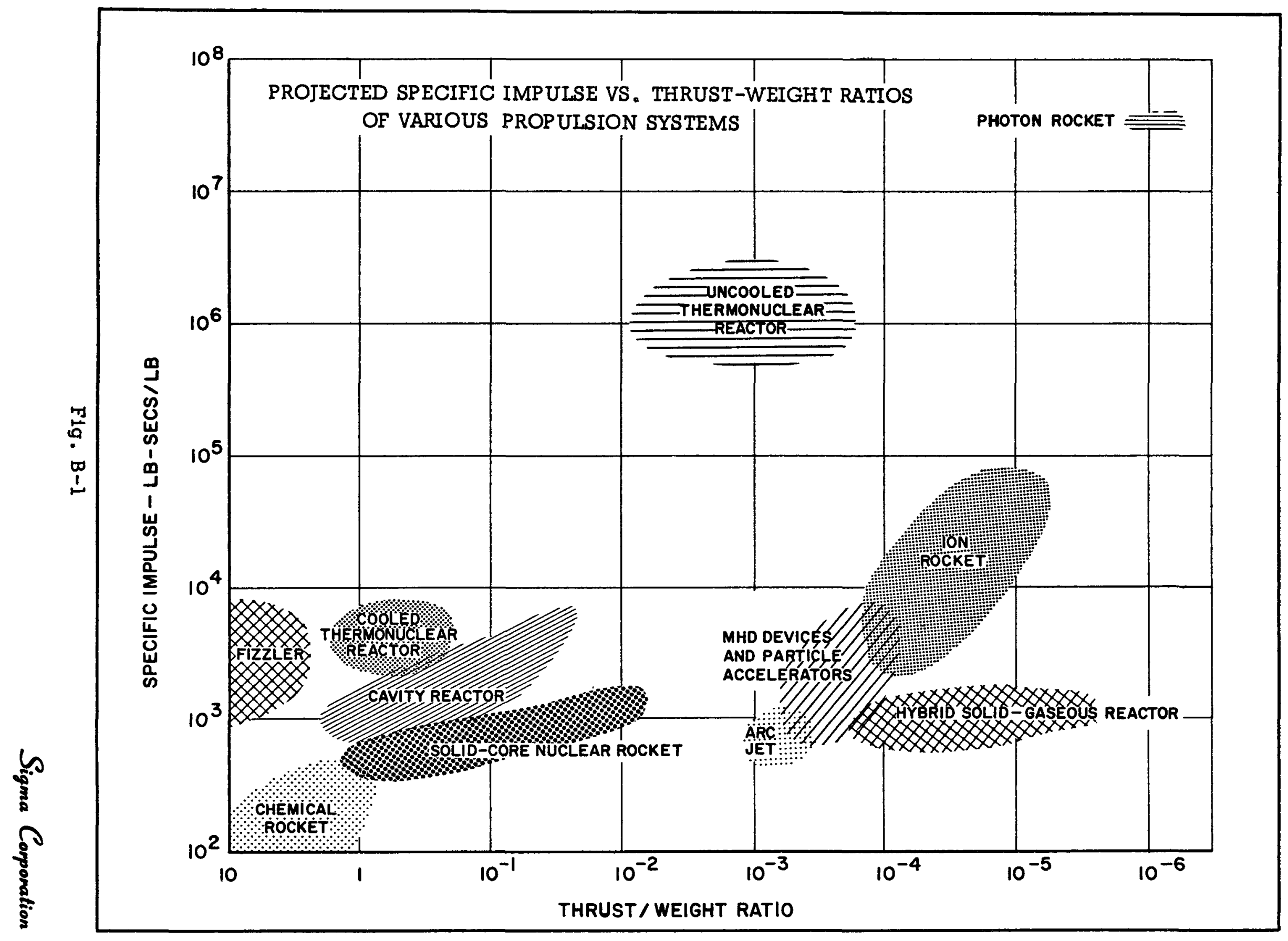




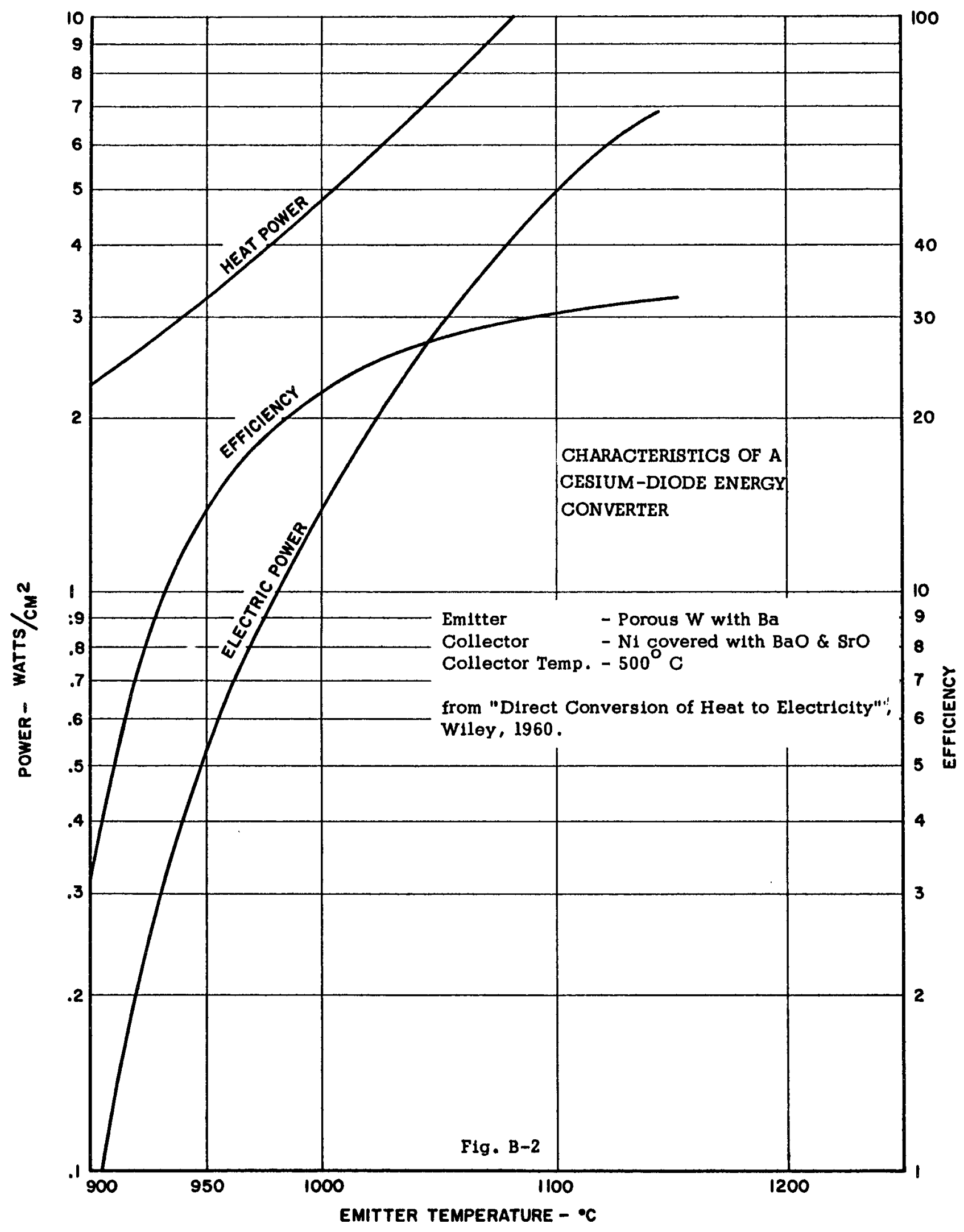


And they offer the possibility of bettering by a factor of two or three the efficiencies obtained with turbo-generators.

It is presumed that any reactor in a nuclear-electric propulsion system will be liquid cooled, but the use of vacuum or cesium diodes suggests the possibility of a design which does not involve liquid cooling and is at least conceptually simple. The essence of the design is that the circular sides of a cylindrical reactor be covered with cesium diodes and that the collector electrodes be cooled by radiation into space. The reactor will heat the emitter electrodes of the diodes by direct conduction, there being no internal coolant. Calculations indicate that there are no great incompatibilities in the area requirements. While this design is conceptually simple, construction of a usable system would be difficult because of the temperatures involved. If such a design is not feasible, then internal cooling of the reactor must be used, and then there is no commanding reason for placing the diodes on the reactor, although this might still be a desirable design feature.

If the diodes are placed on the reactor, in effect integrating the reactor and the conversion stage, the propulsion stage is then pretty much an independent entity. Since there need be only electrical connections between the conversion and propulsion stages such an arrangement is not impractical. It is possible, however, to conceive of locating the propulsion units on the base of the reactor, using the temperatures and/or radiation of the reactor in the ionizing process of an io-propulsion unit, while the out- 
put of the diodes provides the accelerating potential. There is, however, a basic, although probably not insurmountable, incompatibility here because the output of the diodes is in the order of a few volts while the accelerating potential of the propulsion unit shoudl be many times that.

Since it appears that it will be necessary to cool the reactor internally, the possibility of integrating the conversion stage and the propulsion stage exists. The possibility of such an integration is enhanced when one considers the similarities between some of the conversion devices and electric propulsion devices. An outstanding example is the case of the cesium-diode conversion device and the cesium-ion electric propulsion device.

The use of thermionic cesium diodes for production of electrical energy from heat energy in conjunction with cesium-ion propulsion units, which essentially convert electrical energy into directed thermal energy, leads to interesting speculations. In this section it is assumed that the reactor is cooled by a liquid metal which then heats the emitter electrodes of the diodes. After being cooled it may return directly to the reactor. The collector electrodes of the diodes will have to be cooled, and this may be done by radiation into space or by cooling by a liquid metal which is cooled in a radiator. If the latter course is selected, there will be two liquid metal loops, and while it may be possible to combine them into a single loop, calculations will have to be made to determine the better design; it appears, however, that a twoloop system would be better. 
The cesium diode and the cesium propulsion units have some features in common but they also have some unmatched features . They both require cesium ions, and presumably evaporation from a heated tungsten surface would be used in both cases. However, a single diode will develop around a couple volts while a propulsion unit will require voltages around 100 or 1000 volts. However, the diode can develop around 1 to 2 watts $/ \mathrm{cm}^{2}$, which is about the rate the propulsion unit will dissipate electrical energy, so that what is required is (1) a single method whereby the voltage of the diode can be increased, or (2) the propulsion units made to work at lower voltages.

One possible scheme is illustrated in the sketch.

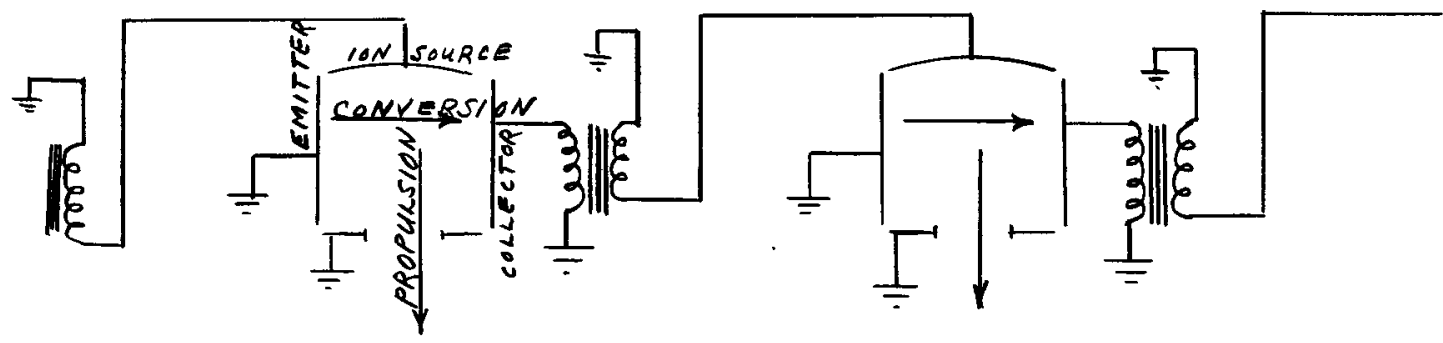

The two combined thermionic diodes and propulsion units shown are considered to be only part of a larger set of the units. When there are cesium ions present in the space between the diode electrodes, the electrons will flow from emitter to collector, but when there are no cesium ions present, the electron flow rate will be essentially zero. The cesium-ion source will be emitting ions continuously, and as the ion concentration builds up, the electron 
flow will increase. If now a voltage is applied in the proper manner across the ion-source and aperture electrodes, the cesium-ions will be ejected through the aperture, resulting in a thrust being applied to the unit. When the cesium ions are ejected the electron flow rate will decrease. This will result in potential drop across the transformer secondary, which will be considerably larger than that across the primary coil, thereby a voltage is applied across the propulsion electrodes of the next unit. The ejection of the ions from this unit will result in a cessation of electron flow in the unit, and a voltage consequently is applied across the propulsion electrodes of a third unit, and so on. Eventually, the last unit of the chain will be connected to the first unit so that a complete circuit is formed.

The above is only a genesis of an idea, but it does appear that a workable system could be devised.

The advantages of this scheme are chiefly due to the intermittent operation of the diode, which results in an alternating current whose voltage can be increased to be more compatible with the propulsion-unit requirements, and the fact that if the diode is to be operated intermittently, the propulsion unit must also be operated intermittently if it is to be tied in closely with the diode unit. It is, of course, not immediately obvious that it is necessary to have such close connections between the conversion units and the propulsion units.

There appears to be one advantage in a pulsed propulsion unit in that neutralization of the ions after ejection may be facilitated. The average thrust of a unit will, of course, be less than 
if it were operated continuously under the same conditions.

In a manner comparable to the cesium diode case, the magnetohydrodynamic thermal energy conversion schemes are similar to some of the magnetohydrodynamic propulsion ideas. However, since both the conversion schemes and the propulsion ideas are so intangible at present, it is difficult to work on the problems of combined systems. Such systems certainly cannot be ruled out of the realm of possibilities.

\section{CONCLUSION}

There is sufficient promise in the wholly or partially integrated systems that they cannot be disregarded and while it may be possible and reasonable to design the reactor as a separate entity, such a course may lead to designs which compare unfavorably with integrated designs. 


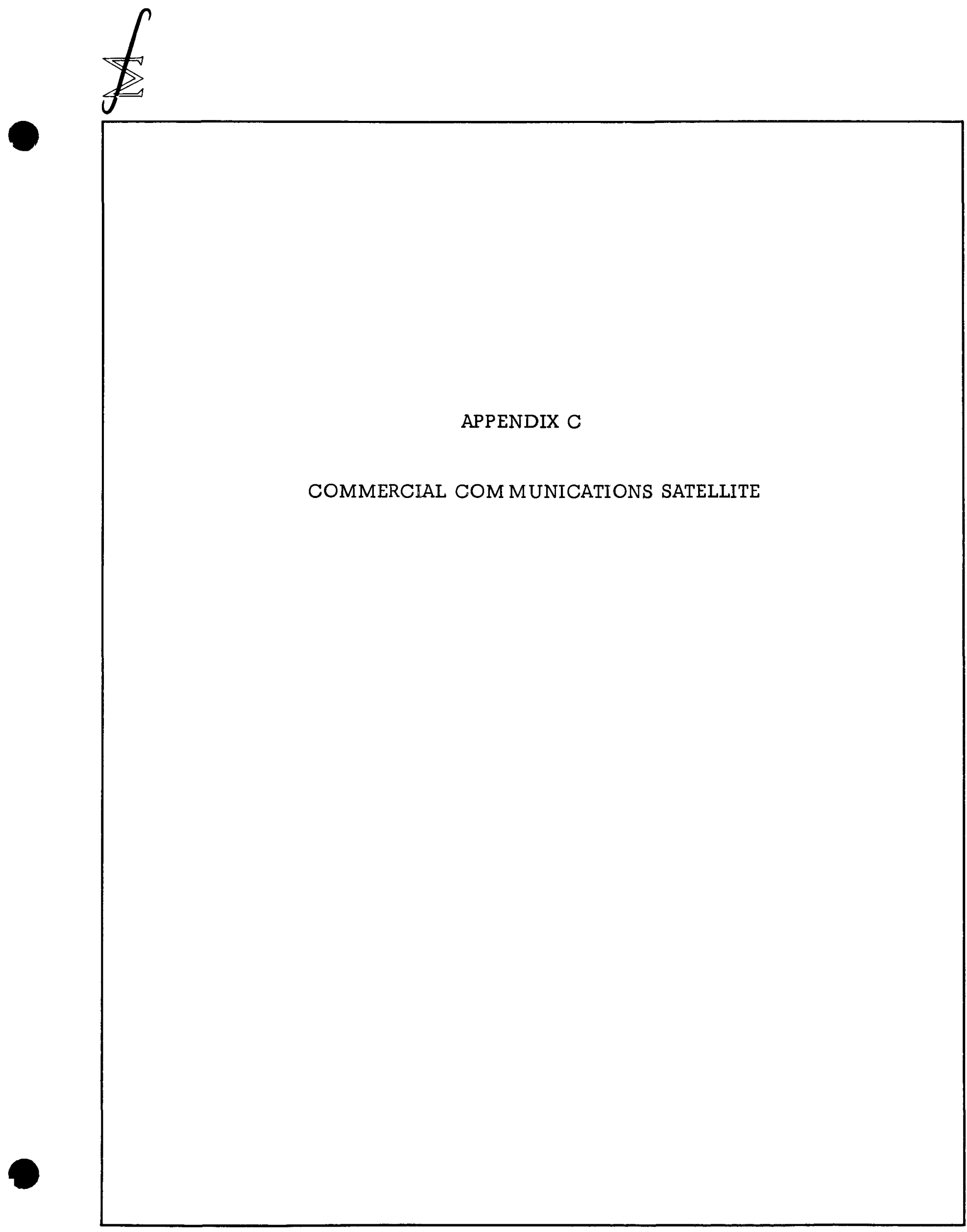

Sigma Corporation 


\section{APPENDIX C \\ COMMERCIAL COMMUNICATIONS SATELLITE}

The concept of using satellite vehicles as relay stations in long distance communication networks has long been studied by scientists and engineers. Until recently these studies have been academic exercises because the basic technology of satellite vehicles, which are the key system component, was not sufficiently advanced to consider an actual hardware program. This situation is, however, rapidly changing and agencies which are in a position to initiate actual programs, as in the case of ARPA, have either initiated hardware programs or in other cases are considering initiating programs. Two somewhat different and interesting examples are given in the following paragraphs: The following is quoted from an article entitled "The Radio Voices of Space" which appeared in the March 1960 issue of the magazine SPACE AGE.

"The Russians have proposed a system of international TV using three stationary satellites (they rotate with the earth) 22,000 miles above the earth. This system, described last year in the Russian magazine RADIO by engineer V. Petrov, calls for each of the three satellites to house elaborate TV receiving equipment and a 10,000 watt TV transmitter. Signals would be transmitted, say, from the U.S. to one satellite overhead. The U.S. satellite would relay the signal to another satellite hovering 45,000 miles away over Russia or China which would then re- 
broadcast the signal back to earth. Elaborate computations were given by Petrov to compensate for the slow drift of the satellites plane around the earth. This whole idea might strike you as being a product of the latter day Jules Verne; however, it should be seriously considered in view of a report which appeared in December 1958 in AFTENPOSTEN, Norway's largest daily newspaper, and also in a bulletin from the Office of Technical Services of the U.S. Department of Commerce." The item went on to present evidence that Russia was experimenting with TV transmissions or relay transmissions from an artificial satellite.

From ELECTRONIC NEWS, May 9, 1960, at an annual Forum of National Aeronautical Electronics conference held in May。Gen. J. T. Bestic, Deputy Chief, Electronics, USAF, discussed military interests in space communications. In his talk, he noted that polar satellites would offer new, fail-safe command and control of SAC aircraft. He also predicted that we are approaching an economically competitive situation in which equatorial satellites can do the job of conventional techniques. As an example, he pointed out that a Pacific cable which is not feasible at the present estimated cost of $\$ 420,000,000$ can soon be replaced more economically by a communication satellite system.

All of this seems to indicate that in the reasonably near future, serious com sat programs will be initiated for commercial purposes and system design teams will be undertaking the design of such systems. As part of this effort, these designers will be 
concerned with the selection of an APU system and will undoubtedly consider the use of SNAP.

At the present time a system engineer concerned with the design of a space system is very much constrained to think in terms of technical feasibility. Many of the key components of his system have technical limitations concerning other parts of the system. This situation results for the most part in the relegation of many economic considerations to a position of secondary importance in overall system design. (This is not to say that the system designer is not aware or not concerned with fundamental economic considerations for he obviously is. The program must be deemed to economically feasible and is funded on the basis of broad economic considerations. We are concerned here with more detailed economic considerations associated with optimization of a system from an economic standpoint.)

As man progresses in the field of space technology as he has in other technological fields, he will undoubtedly find that the systems that he designs must enter into economic competition and that his system design becomes more and more influenced by economic considerations. The economic optimization of a system at this stage is further complicated by the fact that the range of technical choice has been greatly enhanced by technical progress.

That part of the technical progress that is of particular concern at this time is the advent of SNAP units for application to space systems. The particular advantage of SNAP units that is of interest to the system engineer concerned with space systems 
is the potential they offer for a long-lived, reliable and continuous high power source. With the advent of SNAP units, system engineers will find their horizons considerably broadened from what they are now. The technical disadvantages of present APU systems are well-known and need not be particularly discussed. In many cases these disadvantages have been overcome by enhancing the capability of other system components to achieve satisfactory system performance. Sometimes at moderate cost but more often at very high costs indeed. However, the system designer did not have to be greatly concerned by this fact since in general, he had no alternative. Particularly if, as has usually been the case, he had a tight schedule to meet. However, as has been said before, designers of future systems will find themselves more constrained to optimize their system from an economic standpoint and in many cases, the higher power available from SNAP units can be used to advantage in reducing system costs .

In order to illustrate this point, it is probably best to show by specific example how system design considerations from an economic standpoint can be affected by the advent of SNAP units. The example that we have chosen for this demonstration is a possible "single thread" design of a com satellite network. This example is by no means offered as a com satellite system design. The design of a com sat system will be a very complex undertaking and many factors must be taken into account which have not been taken into account in our example, but we do believe that the considerations we have arrived at are valid and do serve to illus- 
trate the economic advantages of a SNAP unit for at least one potential application.

We also feel that this particular application, i.e., a com sat system, is one of the most important applications of satellites to human progress. A commercial com sat network has the potential for not only providing intercontinental wide band communication links which are not now technically feasible but a commercial com sat system also seems to have the potential for competing economically with present intercontinental links such as submarine cables. It has been estimated that conventional cable links cost $\$ 200$ per voice channel mile and that a competing link using present techniques could be installed for $\$ 300$ per voice channel mile. If past history is any indication, system economies will surely be achieved which can combine to reduce the cost of a satellite system well below the $\$ 200$ per voice channel mile given sufficient demand for the large capacity which can be attained. The $\$ 300$ figure quoted was based on 96 channels. At present the capacity of the Atlantic system is only 36 channels but is admittedly inadequate. A single TV link would consume 1,000 voice channels and it seems not unthinkable that at least one TV channel could be utilized even at present for commercial purposes.

Returning to our example of a com sat communications link, Fig. C-1 shows the major components of this link.

In Fig. C-l we have a pictorial representation of a satellite vehicle having a 24-hour orbital period stationed over the 
f

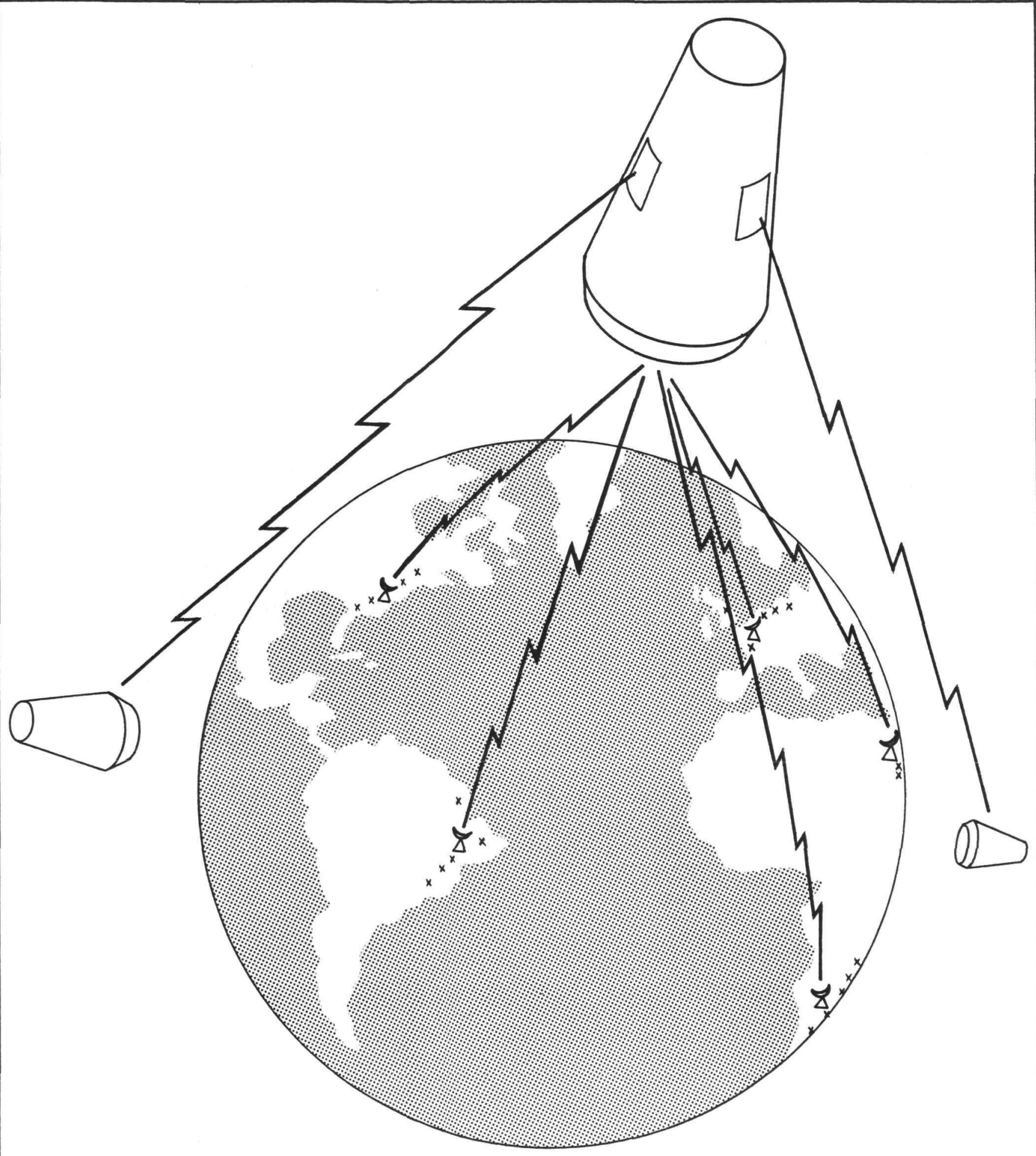

ATLANTIC LEG OF A COMMERCIAL COMMUNICATIONS SATELLITE SYSTEM

Fig. C-1 
Atlantic. Five ground stations located in major population areas use the active repeater aboard the satellite vehicle to link themselves together. These stations, in turn, are associated with a conventional ground distribution system to provide customer service throughout the geographical area served. This satellite is also linked to two other satellites which form the total system of three equally-spaced satellites.

By making a few general and admittedly somewhat arbitrary assumptions, we can arrive at some relationships which allow us to examine the possible advantages of SNAP power units.

Our first basic assumption is the use of the 24-hour orbit. Systems based on the use of lower orbit altitudes have been and must continue to be examined. However, a system based on the 24 -hour orbit is generally considered to be a promising possibility.

Our second assumption concerns the selection of a frequency. It is generally agreed that near optimum frequencies for earth to space communications lay between 300 and 3,000 megacycles. At lower frequencies ionospheric effects are troublesome; at higher frequencies atmospheric absorption is a limiting quantity. We have, therefore, arbitrarily selected 1,700 megacycles as a probable frequency as an existing authorized frequency for this purpose.

Given these basic assumptions and bearing in mind that such a commercial enterprise as this Com Sat system is must be extremely conscious of economic factors, we are now in a posi- 
tion to examine the effect of satellite transmitter power on ground station cost.

Fig. C-2 illustrates the relation of radiated power of the satellite transmitter to the ground station antenna cost which in the extreme case of a very large antenna, is the major factor in the station cost. The three different antenna gains have been selected on the basis of the complexity of the satellite attitude stabilization requirements.

At $0 \mathrm{db}$ gain, of course, there is no requirement for attitude stabilization. At $3 \mathrm{db}$ gain, we illustrate the requirement for only the simplest type of attitude stabilization to keep the vehicle pointed only generally in the direction of the earth. At 16db gain and greater, the vehicle must be stabilized within a few degrees at all times.

From the chart we can see that the case of very low power introduces a high cost factor for ground station installations. However, moderate ground station costs do appear to be achievable with the case of moderate power for a stabilized vehicle and with unstabilized vehicles for power requirements of the order of those available with SNAP 2. The radiated power shown here must, of course, be multiplied by some factor such as 5 to arrive at an approximation of the total APU requirements. Then for a basic antenna cost of $\$ 50,000$, we have a 3,000 watt APU requirement for an unstabilized vehicle and a 50 watt plus stabilization requirement for a stabilized vehicle.

The foregoing considerations assume a vehicle capability 


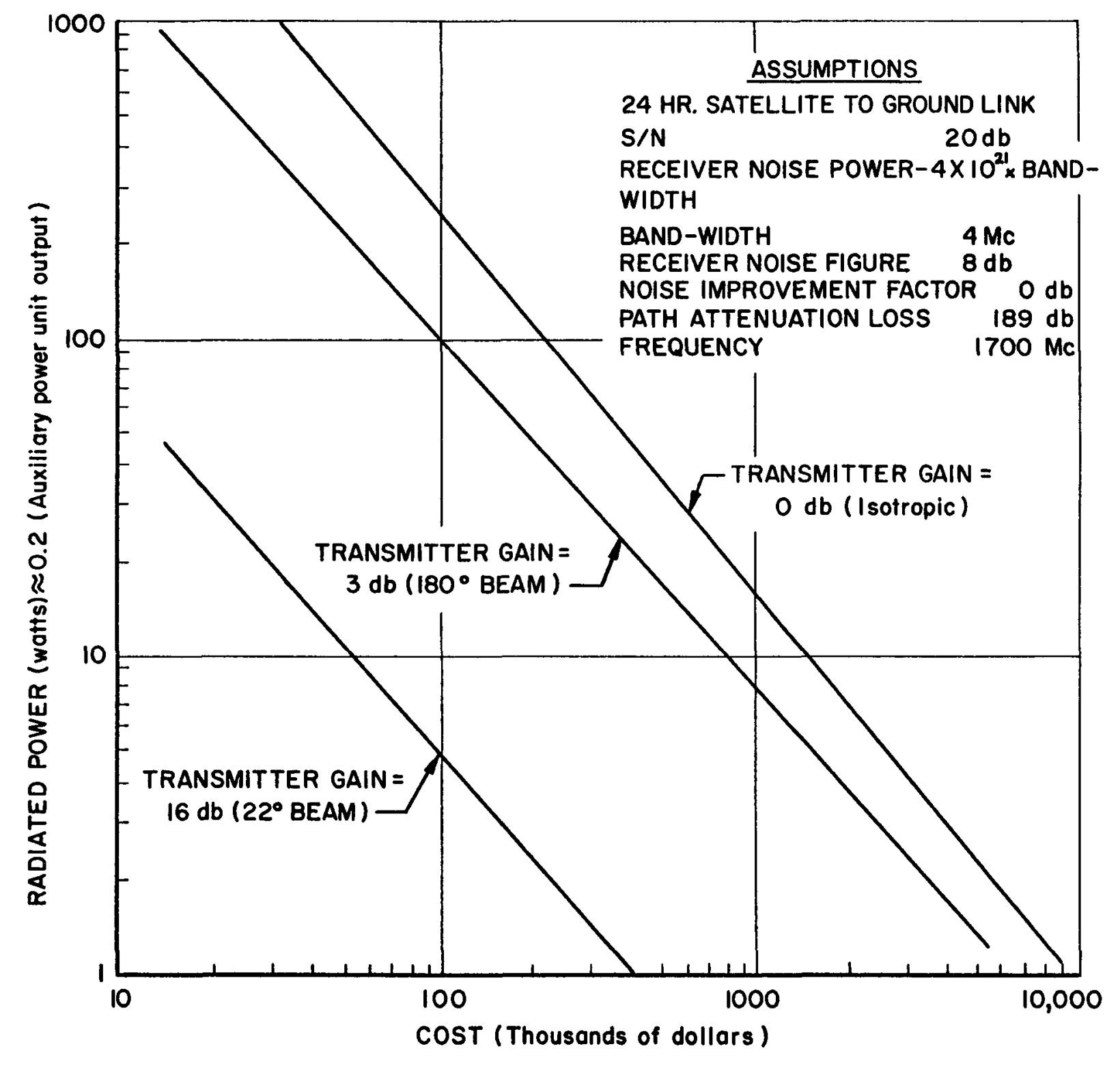

TRAINABLE ANTENNA COST FOR GROUND

INSTALIATION VS. RADIATED POWER

Fig. C-2 
for 300 voice channels and 1 video channel (Bandwidth $4 \mathrm{mc}$ ). However, if past history is any indication, this system will open new markets for communication services with an attendant increase in bandwidth requirements which must be compensated for by enlarging the ground station antenna, increased vehicle stabilization and greater transmitting antenna gain or an increase in the vehicle power with the increase in vehicle power being most probably the least costly of the alternatives. 